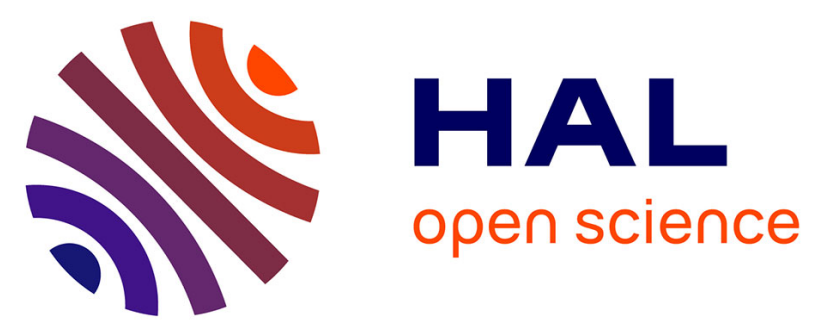

\title{
Large axisymmetric surface deformation and dewetting in the flow above a rotating disk in a cylindrical tank: spin-up and permanent regimes
}

\author{
Wen Yang, Ivan Delbende, Yann Fraigneau, Laurent Martin Witkowski
}

\section{- To cite this version:}

Wen Yang, Ivan Delbende, Yann Fraigneau, Laurent Martin Witkowski. Large axisymmetric surface deformation and dewetting in the flow above a rotating disk in a cylindrical tank: spin-up and permanent regimes. Physical Review Fluids, 2020, 5, pp.1-27. 10.1103/PhysRevFluids.5.044801. hal-02937946

\author{
HAL Id: hal-02937946 \\ https://hal.science/hal-02937946
}

Submitted on 30 Oct 2020

HAL is a multi-disciplinary open access archive for the deposit and dissemination of scientific research documents, whether they are published or not. The documents may come from teaching and research institutions in France or abroad, or from public or private research centers.
L'archive ouverte pluridisciplinaire HAL, est destinée au dépôt et à la diffusion de documents scientifiques de niveau recherche, publiés ou non, émanant des établissements d'enseignement et de recherche français ou étrangers, des laboratoires publics ou privés. 


\title{
Large axisymmetric surface deformation and dewetting in the flow above a rotating disk in a cylindrical tank: spin-up and permanent regimes
}

\author{
Wen Yang, ${ }^{1}$ Ivan Delbende, ${ }^{2}$ Yann Fraigneau, ${ }^{3}$ and Laurent Martin Witkowski ${ }^{2, *}$ \\ ${ }^{1}$ Sorbonne Université, Collège Doctoral, F-75005 Paris, France ${ }^{\dagger}$ \\ ${ }^{2}$ Sorbonne Université, Faculté des Sciences et Ingénierie, \\ UFR d'Ingénierie, F-75005 Paris $\ddagger$ \\ ${ }^{3}$ LIMSI-CNRS, Bât 507, rue du Belvédère, F-91405 Orsay Cedex, France
}

(Dated: February 11, 2020) 


\begin{abstract}
In this paper, we aim at characterizing the spin-up process and the permanent regime of a rotating flow with free surface. The motion is created by the quasi-impulsive rotation of a disk located at the bottom of a cylindrical tank partially filled with a Newtonian water-glycerol mixture at a fixed initial aspect ratio (height to radius) of 0.25 . Two experimental setups with different radius sizes and two numerical codes with distinct formulations are used, allowing for numerous comparisons and cross validations. Time-dependent surface height profiles and azimuthal velocity profiles are accurately measured with a laser line sensor and Laser Doppler Velocimetry respectively. These measurements are compared to numerical results showing an overall excellent agreement and whenever a discrepancy exists, explanations are given. The spin-up and permanent regime for two typical flow cases are detailed: $(i)$ one for which the angular speed moderately deforms the free surface and (ii) one at higher angular speed for which the disk becomes partially dewetted. The shape of the free surface in the permanent regime for intermediate rotation speeds is also reported. The flow parameters are chosen in such a way that large deformations of the free surface are achieved, yet remaining under the threshold of appearance of rotating polygonal patterns. This enables us to characterize realistic base flows on which this instability may develop.
\end{abstract}

\title{
I. INTRODUCTION
}

A disk rotating at the bottom of a cylindrical cavity partially filled with an homogeneous Newtonian fluid can generate instability patterns that have attracted the interest of the community. Some of these patterns observed in experiments take the form of rotating polygons that break the initial axisymmetry of the free surface [1-8]. Depending on the initial liquid height and disk angular speed, ellipse, triangle, square, pentagon and hexagon polygon-like patterns are formed, which rotate in the same direction as the disk. These rotating polygons (RP) have been ascribed to a resonance between gravity and centrifugal waves, and manifest themselves as a strong nonaxisymmetric deviation of the free surface height.

* laurent.martin_witkowski@sorbonne-universite.fr

† Also at Laboratoire FAST, CNRS, Université Paris-Sud, Université Paris-Saclay, 91405 Orsay, France;

Now at Sino-European Institute of Aviation Engineering, Civil Aviation University of China, 300300

Tianjin, China

¥ Also at LIMSI-CNRS, Bât 507, rue du Belvédère22F-91405 Orsay Cedex, France 
Another instability, hereafter called bulk-driven instability (BI), has been observed for lower angular speed. While RP are clearly associated to large amplitude surface waves, BI barely deforms the interface from axisymmetry. Thus, BI can only be visualized by seeding the fluid with flakes/injecting dye or measured by Laser Doppler Velocimetry (LDV)/Particle Image Velocimetry (PIV) [9-12].

These two types of instability have been studied separately by different research groups. BI were first visually detected by Spohn et al. [9] while studying vortex breakdown at different aspect ratios (defined as the fluid height-to-radius ratio at rest). As the disk angular speed was increased, they could evaluate the instability threshold when the recirculation bubble starts oscillating. This threshold value was later confirmed and refined by Young et al. [10] thanks to LDV measurements. Hirsa et al. [11] were the first to characterize the azimuthal structure of these modes in the horizontal plane using PIV. For an aspect ratio of 2 , they observed a rotating mode of azimuthal structure $m=4$, later confirmed by numerical simulations [13]. Poncet \& Chauve [12] experimentally explored BI at aspect ratios much lower than 1 . They revealed that the saturated instability takes the form of several co-rotating vortices evenly distributed along a circle, with an azimuthal wavenumber increasing as the aspect ratio is decreased. For the same configuration, Kahouadji et al. [14] numerically described the base flow and performed its linear stability analysis, confirming this trend.

Concerning RP, the description of the flow on which the instability develops is quite recent $[4,15,16]$. These descriptions are based on analytical models. Despite necessarily strong assumptions, some of these models have proven to successfully predict the free surface deformation reported in experiments [17] or to understand the occurrence of RP in terms of wave resonance [18]. None of these studies address the existence of BI.

Numerical simulations of RP are not as widespread as they face many challenges. Large surface deformation, interface with small air-to-liquid density ratio, thin boundary layers, complex dynamics of the contact line, dewetting of the disk are among the difficulties that have to be overcome. To our knowledge, no numerical simulation has been carried out in this configuration taking into account all these constraints. Order one density-ratio simulations have been performed for instance by Brady et al. [19] and Carrion et al. [20], the latter reference including a comparison with the experimental data obtained by Fujimoto et al. [21]. Other simulations have been carried out with small density ratio [22, 23]. In [23], 
the surface tension effects are small enough so that the interface shape could be compared successfully to an experiment close to the configuration where RP can develop. However, disk dewetting has not been tackled in these studies and, except in [19], temporal evolution is not reported.

In a previous paper [24], we numerically described the mean base flow at large Reynolds numbers, yet focusing mainly on the flat surface case to circumvent the difficulty of 3D twophase flow numerical simulations. In the present paper, we investigate another parameter range where surface deformations are large and Reynolds numbers are kept moderate. This choice enabled us to perform reliable numerical simulations of the flow below RP thresholds. Experiments are tailored to fit this regime. Free surface height profiles and local azimuthal velocity measurements are carefully compared to numerical simulations in both steady states and transient regimes. We mainly present results for an aspect ratio of 0.25 , for which $\mathrm{RP}$ ranging from ellipse to hexagon have been observed $[2,5]$.

A long-term goal is to simulate RP, it is thus necessary to ensure that the simulations can accurately reproduce not only the steady states but also temporal evolution of the free surface. Indeed, the experimental protocol may influence the phase diagrams identifying RP. Unfortunately, the protocol is not always reported but it is common practice to gradually increase the angular speed of the disk. As pointed out by Bach et al. [6], different protocols can lead to different states and for a fixed filling height, hysteresis can occur when the angular speed increases and then decreases. High-fidelity simulations must follow the same protocol as in experiments in order to reproduce the spin-up (or spin-down) process precisely.

Spin-up is a well known subject in the rotating fluid community. Two reviews [25, 26] outline important results on the spin-up of homogeneous fluids completely filling closed containers. We summarize here some key points also relevant to understand the dynamics of the flow with a fixed side wall. When the whole container is rotating as a single unit, the fluid inside eventually reaches solid-body rotation independently of its initial state. The torque applied by the faster walls to the slower fluid vanishes in the steady state where the angular speed becomes uniform. Most of the early work focus on the linear spin-up for which a small speed increment is applied to the container where the fluid has already reached solid-body rotation. This regime can be described within the framework of the linearized Navier-Stokes equations. The fluid angular speed adjustment is not a diffusive process but results from angular momentum transport through a transient meridional flow. 
An unbalanced centrifugal pressure gradient generates a flow directed radially outward in a thin boundary layer close to the bottom (and top) disk. The fluid rotating slower than the disk is sucked by continuity into the disk boundary layer and accelerated (Ekman pumping). The path is completed along the side wall before returning to the bulk. By doing so, the bulk gains some angular momentum and this entire process stops when the angular velocity deficit between the bulk and the walls fades away.

When the speed increment of the container is of the same order of magnitude as the initial speed of the fluid, the linearisation of the Navier-Stokes equations no longer holds. This is typically the case when the fluid is spun-up from rest. Solutions of the nonlinear spinup are also discussed in the reviews and are developed so as to achieve better agreement with experiments [27-29]. Simulations of this problem were also conducted to assess the theoretical and experimental work $[30,31]$ and were able to exhibit weak inertial oscillations of the nonlinear regime.

Compared to the closed container, very limited studies include the effect of the free surface on the spin-up. Greenspan et al. [32] studied the effect of free surface in the linear regime. It was extended to the nonlinear regime by O'Donnell et al. [33]. If surface-tension effects are weak, the free surface asymptotically reaches the well-known parabolic shape. The main effect of the free surface is to introduce another dimensionless parameter, namely the Froude number. A moderate correction to the Ekman timescale for the spin-up process has been proposed for large Froude numbers [33]. Numerical simulations of Navier-Stokes equations in both air and liquid phases without using a semi-analytical model have been undertaken by Yan et al. [34] and can be considered as a pioneering numerical work on this system.

In the present configuration, the side wall is fixed, so that the flow cannot reach solid-body rotation. The regime is nonlinear as the azimuthal velocity variation always remains order one. Nevertheless the key ideas behind the Ekman pumping mechanism are still relevant and the meridional recirculation plays a major role. An important difference lies in the fact that the torque applied by the rotating disk to accelerate the fluid never vanishes, and a steady state is reached when this torque is exactly balanced by the opposite torque applied by the side wall decelerating the fluid. The previously mentioned analytical models $[16,35]$ rely on estimations of these torques.

The present study proposes an alternative approach by solving the full Navier-Stokes equations for the free-surface flow. Steady and unsteady solutions are compared to ex- 
perimental results. The structure of the paper is as follows. In section II, experimental techniques are presented, as well as the governing equations, the dimensionless parameters and a brief overview of the numerical codes and methods used. Transient and permanent regimes of one case at moderate disk speed, where no disk dewetting occurs, are presented in section III, including a discussion on the potential influence of bulk instabilities on the shape of the free surface. Section IV then describes the evolution of the asymptotic permanent regime as the disk speed is increased into states where disk dewetting occurs. Spin-up and permanent flow at large disk speeds are presented in section $\mathrm{V}$, which eventually focuses on the influence of disk dewetting on the flow field and on the azimuthal velocity distributions. Conclusions are given in section VI. Details on the numerical technique used for unsteady two-phase flow simulations are presented in the Appendix.

\section{CONFIGURATION AND METHODS}

\section{A. Experimental methods}

\section{Experimental setups}

Two experimental setups of different sizes have been used. Both correspond to the same configuration shown schematically in figure 1(a). The cylindrical tank of radius $R$ is filled at an initial height $H$ with a Newtonian liquid of density $\rho_{1}$ and dynamic viscosity $\mu_{\mathrm{l}}$. A disk located at the bottom of the tank rotates at the angular speed $\Omega$. The characteristics of both setups are listed in table I. The large tank with a radius of $140 \mathrm{~mm}$ is exactly the one used in [24] while the small one with a radius of $70 \mathrm{~mm}$ is designed to explore a larger range of parameter values. Both large and small setups can be mounted on the experiment table so that their respective bottom disks are driven by the same DC brushed motor with a tachometer closed-loop speed control.

The DC motor model is a RX 320E produced by Parvex. It is mounted directly to the shaft of the disk with a gearbox reduction of 12 to 1 . The speed loop control is achieved using a Parvex servo amplifier (RTS model). This servo amplifier that drives the DC motor has an input voltage between 0 and $10 \mathrm{~V}$ provided by the analog output of a micro-controller card Mbed FRDM-KL25Z, amplified with a gain of 3 to reach the desired amplitude.

Height variations of both disks were checked by measuring locally the displacement of 


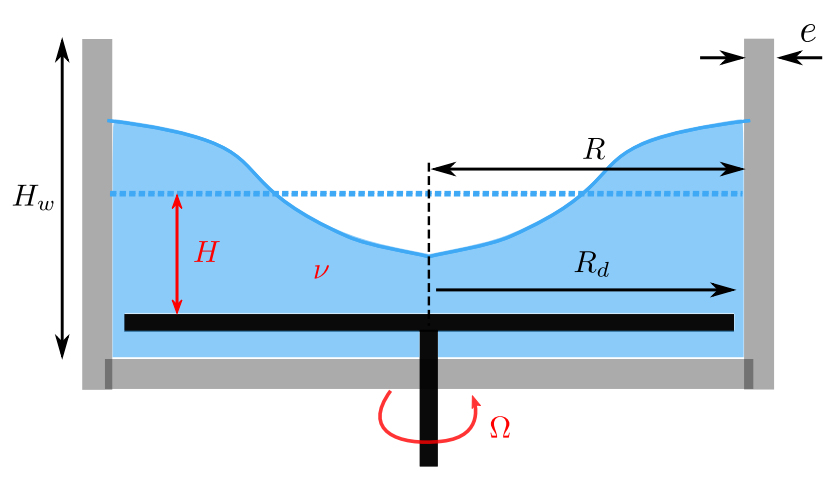

FIG. 1. Scheme of the experimental setup.

\begin{tabular}{lcc} 
Setup & $\mathrm{L}$ & $\mathrm{S}$ \\
\hline Cylinder inner radius $R(\mathrm{~mm})$ & 140 & 70 \\
Disk-wall gap $R-R_{d}(\mathrm{~mm})$ & 0.7 & 2 \\
Cylinder wall thickness $e(\mathrm{~mm})$ & 6.8 & 5.6 \\
Side wall height from disk $H_{w}(\mathrm{~mm})$ & 71.5 & 114 \\
Cylinder wall material & PMMA & glass \\
\hline
\end{tabular}

TABLE I. Characteristics of two experimental setups in laboratory.

their upper surface while being rotated at different angular speeds. We observed a maximal amplitude of $0.15 \mathrm{~mm}$ for the large disk and $0.2 \mathrm{~mm}$ for the small disk. The temporal analysis of the local height variation signal shows that the spectra for both devices are localized around the disk rotating frequencies.

A mixture of $80 \mathrm{wt} \%$ glycerol and $20 \mathrm{wt} \%$ water has been used in most experiments of the present study and is denoted as G8W2 in the following. G8W2 has a dynamic viscosity of 61 times that of water at $T=20^{\circ} \mathrm{C}[36]$.

\section{Measurements}

In order to characterize the flow in the transient regime from the start of the bottom disk rotation, we have measured the surface height $h(r, t)$ and the azimuthal velocity $V_{\theta}(t)$ at some probe locations.

A laser line profile sensor Gocator 2350 produced by LMI is installed above the fluid to measure the surface deformation along a diameter, with an acquisition rate of $100 \mathrm{~Hz}$, as 


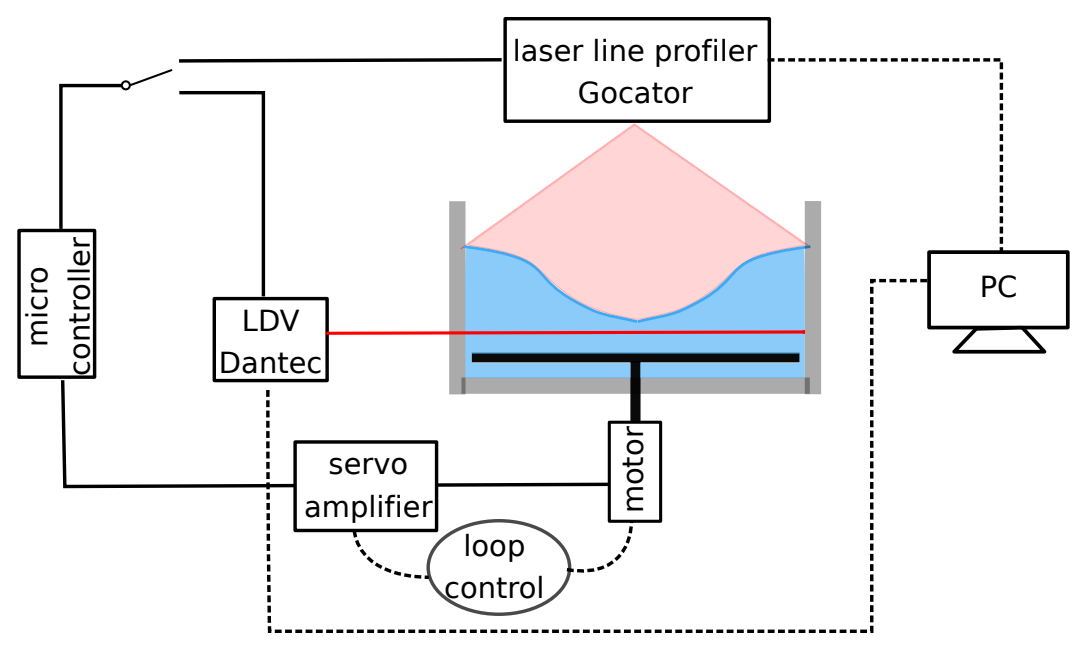

FIG. 2. Sketch of the experimental setup with the control and measuring systems.

shown in figure 2 . The horizontal resolution is approximately $0.25 \mathrm{~mm}$ while the theoretical vertical resolution is below $0.06 \mathrm{~mm}$. Since non-transparency is needed for this laser technique, titanium dioxide particles (Kronos 1002) are used at a concentration of $4 \mathrm{~g} / \mathrm{L}$ to obtain a diffusive reflection without significantly changing the fluid properties [37]. In practice, the precision of height measurements on this white liquid surface drops down to about $0.2 \mathrm{~mm}$, which is quite satisfactory in the present context. We have also verified for some experiments that mass conservation is satisfied. Based on the time-averaged measured height profile $\bar{h}(r)$, one can define the volume error as:

$$
\Delta V=2 \pi \int_{0}^{R} r[\bar{h}(r)-H] \mathrm{d} r .
$$

The obtained volume error corresponds to a height error $\Delta h=\Delta V /\left(\pi R^{2}\right)$, which is about $0.15 \mathrm{~mm}$ in the large setup and $0.06 \mathrm{~mm}$ in the small one.

The evolution of the azimuthal velocity component $V_{\theta}(t)$ at a given probe $\left(r_{0}, z_{0}\right)$ is measured by Laser Doppler Velocimeter (LDV) using the same Dantec BSA system as in [24]. With the help of a horizontal displacement platform and a vertical one, any position in the fluid can be reached. LDV requires a transparent liquid to detect the seeded particles (hollow silver coated glass spheres). Consequently, the measurements of the surface height and flow velocities cannot be conducted at the same time. We use a digital thermometer to monitor the liquid temperature. 


\section{Experimental protocol}

In order to investigate the transient fluid motion from rest, the disk motion and the measurement device are synchronized thanks to a micro-controller card. A TTL (TransistorTransistor Logic) signal is sent either to the laser line profile sensor or to the LDV device, and an analog voltage signal is supplied to the servo amplifier. Figure 2 schematically shows the measuring system.

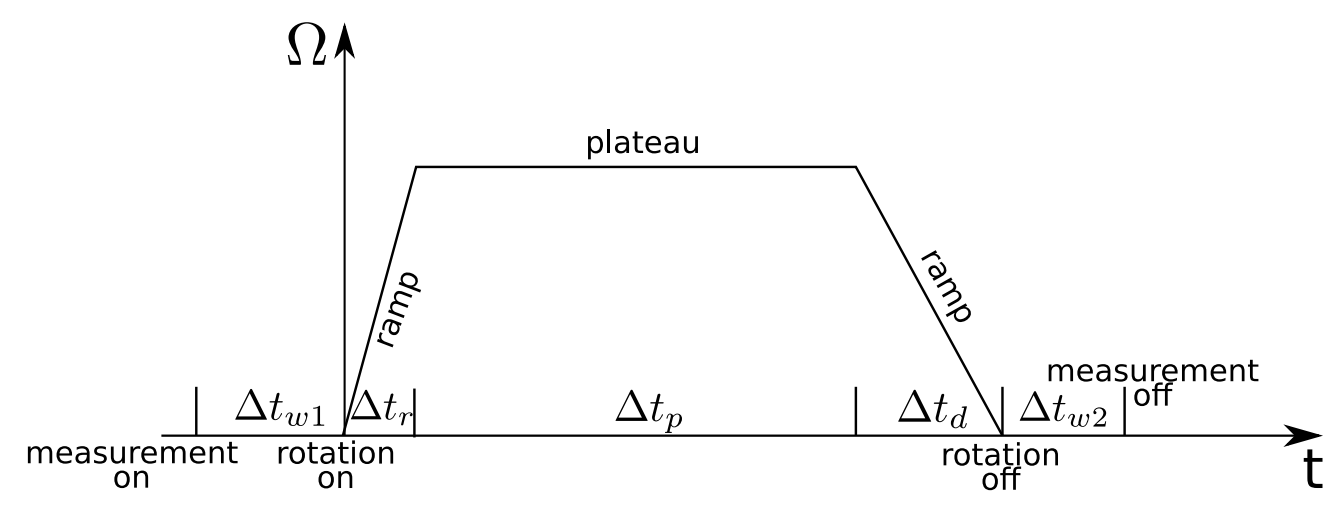

FIG. 3. Schematic representation of the different time constants used in the experimental protocol.

In experiments, the measurement and the disk rotation follow the time protocol shown in figure 3. The measurement is started $\Delta t_{w 1}$ seconds earlier than the start of disk rotation and lasts $\Delta t_{w 2}$ seconds more after the complete stop of disk rotation. The disk is accelerated from rest up to the desired speed within $\Delta t_{r}$ seconds and after a plateau of $\Delta t_{p}$ seconds stopped within $\Delta t_{d}$ seconds. All speed variations are linear in time. The values used for typical experiments are the following: $\Delta t_{r}=0.5$ or $1 \mathrm{~s}, \Delta t_{p}=60$ to $240 \mathrm{~s}, \Delta t_{d}=10 \mathrm{~s}$, $\Delta t_{w 1}=5 \mathrm{~s}, \Delta t_{w 2}=5$ or $10 \mathrm{~s}$. The order of magnitude of these time values are much larger than the temporal resolution achieved by the micro-controller.

\section{B. Equations, parameters and numerical methods}

The fluids are assumed immiscible, isothermal and incompressible. The liquid and the gas phase (air) have densities $\rho_{\mathrm{l}}, \rho_{\text {air }}$ and dynamic viscosities $\mu_{\mathrm{l}}, \mu_{\text {air }}$. Using the liquid density $\rho_{\mathrm{l}}$, the disk radius $R$ and its plateau angular speed $\Omega$ as reference scales, the nondimensional 
governing mass and momentum equations read :

$$
\begin{aligned}
\nabla \cdot \boldsymbol{V} & =0 \\
\rho\left[\frac{\partial \boldsymbol{V}}{\partial t}+(\boldsymbol{V} \cdot \nabla) \boldsymbol{V}\right] & =\frac{1}{R e} \nabla \cdot\left[\mu\left(\nabla \boldsymbol{V}+\nabla \boldsymbol{V}^{T}\right)\right]-\nabla p-\frac{1}{F r} \rho \boldsymbol{e}_{z}+\frac{1}{W e} \kappa \delta_{S} \boldsymbol{n},
\end{aligned}
$$

where the dimensionless density $\rho$ and viscosity $\mu$ are respectively equal to $\rho_{\text {air }} / \rho_{\mathrm{l}}$ and $\mu_{\text {air }} / \mu_{\mathrm{l}}$ in the gas, versus 1 in the liquid. Other quantities in (2) are $p$ the pressure, $\boldsymbol{V}$ the velocity field, $\boldsymbol{e}_{z}$ the vertical ascending unit vector, $\kappa$ the curvature of the free surface, $\delta_{S}$ the Dirac function along the direction $\boldsymbol{n}$ normal to the interface. The Reynolds, Froude and Weber numbers are defined by

$$
R e=\frac{\rho_{\mathrm{l}} \Omega R^{2}}{\mu_{\mathrm{l}}}, \quad F r=\frac{R \Omega^{2}}{g}, \quad W e=\frac{\rho_{\mathrm{l}} \Omega^{2} R^{3}}{\sigma} .
$$

As two fluids are considered, namely air and a glycerol-water mixture, the system is also characterized by the two ratios $\rho_{\text {air }} / \rho_{\mathrm{l}}$ and $\mu_{\text {air }} / \mu_{\mathrm{l}}$.

We use the cylindrical coordinate system $(r, \theta, z)$ with the origin located at the center of the rotating disk. In the axisymmetric framework, the Stokes streamfunction $\psi(r, z)$ defined by

$$
V_{r}=\frac{1}{r} \frac{\partial \psi}{\partial z}, \quad V_{z}=-\frac{1}{r} \frac{\partial \psi}{\partial r}
$$

is convenient to characterize the meridional flow.

Two different in-house numerical codes are used in the present study. The first one called Rose specifically computes axisymmetric steady solutions in the liquid only, using a bodyfitted grid with a free-slip boundary condition at the free surface and no-slip at the walls. The contact angle of the liquid at the side wall is imposed to be $90^{\circ}$. More details on this code can be found in $[23,24]$. The second one called SunfluidH is a more general purpose time-stepper code for two-fluid flows, used here in the axisymmetric framework.

We hereafter present a brief summary of the main numerical methods used by SunFLUIDH to solve the governing equations. At each time step, the mass density and dynamic viscosity variations are first solved, using a level-set method. The distance function equation as well as the equation in the redistancing method is discretized in space using a 5th order WENO scheme and in time using a 3rd order Runge-Kutta scheme. The velocity components are then solved by means of a projection method, in the incremental form proposed by Goda [38]. This method involves an estimation step using Navier-Stokes equations and a correction step 
using a Poisson equation. These equations are discretized with a 2nd order centered scheme in space on staggered grids. The time discretization of the Navier-Stokes equations is carried out with a 2nd order backward differentiation formula. Viscous terms are implicited and solved using an ADI method. The Poisson equation uses successive over-relaxations coupled with a multigrid solver which increases the convergence speed. The reader can refer to the Appendix for details as well as for the boundary conditions related to the complete equation set.

\section{SPIN-UP AND PERMANENT REGIMES WITHOUT DISK DEWETTING}

In this first case, the disk is rotated at a speed level large enough to induce a significant free surface deformation, and yet sufficiently moderate so that the deformation amplitude is kept smaller than the initial liquid height: the rotating disk thus remains always wetted.

The large experimental setup is used, with an initial G8W2 layer of depth $H=35 \mathrm{~mm}$, which corresponds to an aspect ratio $G=0.25$. The disk is uniformly accelerated from rest during $\Delta t_{r}=0.5 \mathrm{~s}$ to reach the plateau speed $\Omega=7.84 \mathrm{rad} / \mathrm{s}$. At a measured temperature of $21.3^{\circ} \mathrm{C}$, the values of the dimensionless parameters are then $\mathrm{Fr}=0.88, \operatorname{Re}=3304$ and $W e=3151$.

Numerically, the same case is simulated using the SunfluIDH code. The computational domain $(r, z) \in[0,1] \times[0,0.5]$ is meshed by $128 \times 64$ cells (except stated otherwise). We have checked convergence using a refined grid of $256 \times 128$ cells. The results are not significantly modified in the liquid and are almost identical to those obtained using RosE (see section III B). However, in some cases, the velocity field in the gas may still depend on the resolution: this is discussed in section VB. Initially, the interface between the air and the liquid is set to $z=0.25$. A time step $\delta t=1.7 \times 10^{-3}$ is imposed, which corresponds to a Courant-Friedrich-Lewy (CFL) number of 0.2. In the numerical simulation, special care was taken to follow the experimental protocol, particularly during the uniform disk acceleration. 
(a)

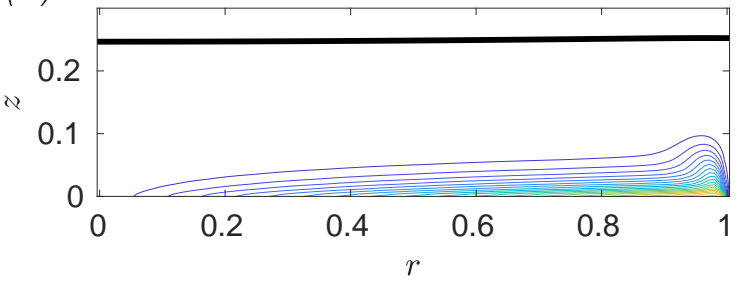

(c)

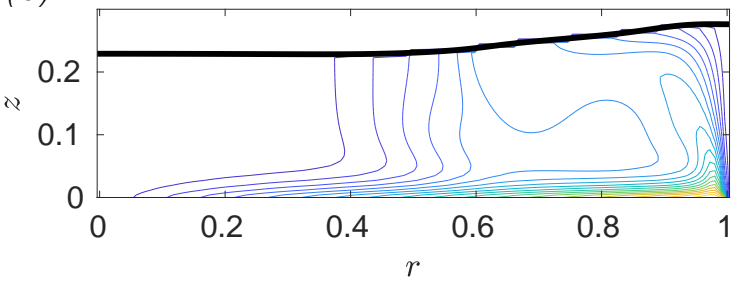

(e)

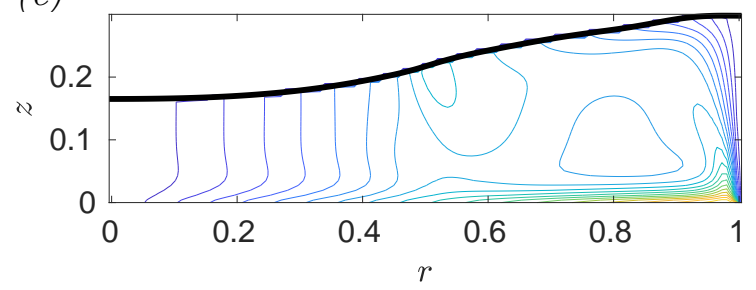

(b)

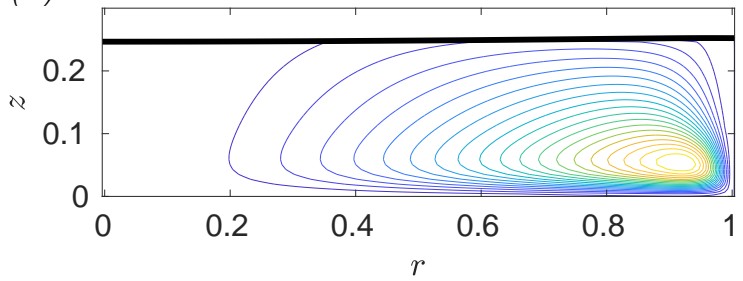

(d)

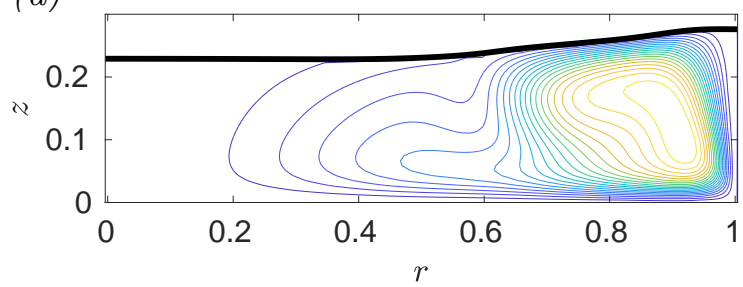

(f)

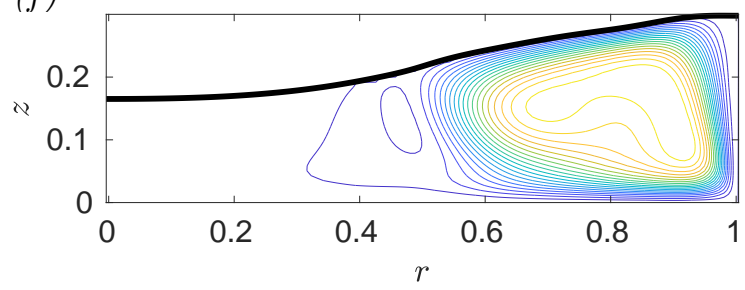

FIG. 4. Snapshots of the flow field in the liquid phase from top to bottom at $t=3.92,23.52$ and 47.04 (dimensional time $t=0.5 \mathrm{~s}, 3 \mathrm{~s}$ and $6 \mathrm{~s}$ ) during the spin-up of the experiment for the case $G=0.25, F r=0.88$ and $R e=3304:$ large setup at $\Omega=7.84 \mathrm{rad} / \mathrm{s}$ and $H=35 \mathrm{~mm}$. (a,c,e): iso-contours of $V_{\theta}$ with 21 equispaced contours between 0 and 1. (b,d,f): Streamlines of the meridional circulation consisting of iso-contours of $\psi$ with 21 equispaced levels between 0 and $\psi_{\max }$ (in the liquid phase), $\psi_{\max }=0.003,0.053,0.056$, respectively. The black lines in all the graphs represent the numerical air-liquid interface.

\section{A. Spin-up}

The spin-up implies a strong coupling between the azimuthal velocity and the meridional flow. This is illustrated in figure 4 displaying specific snapshots of the azimuthal velocity $V_{\theta}$ and the streamfunction $\psi$ in a meridional plane. Short after the disk starts to rotate, angular momentum is at first confined to a narrow layer close to the disk (fig 4 (a)) and the interface remains flat. The meridional flow is maximum near the disk edge but is not intense enough to efficiently convect angular momentum (fig 4 (b)). This diffusive process is rapidly superseded by convection of angular momentum at the periphery $(r>0.5)$ as 
the meridional flow is enhanced through Ekman pumping and spreads out (fig 4 (c,d)). At this stage, the core region $(r<0.5)$ is not yet rotating since the recirculating flow mainly remains in the peripheral region. The spin-up of the core region is a slower process. As it is seen in (fig $4(\mathrm{e}, \mathrm{f})$ ), while the structure of the meridional flow is almost that of the steady state (shown later in fig. 8), the solid body rotation sets in very gradually (the movie of the entire spin-up is provided in the supplementary material) [39]. Whereas the axial pressure distribution is mostly hydrostatic, the radial distribution is directly linked to the azimuthal velocity. The interface thus remains flat for $r<0.4$ up to $t \approx 3 s$ as $V_{\theta} \approx 0$ in the core (fig. 4(c)). It gradually reaches its final shape on the largest time scale.

(a)

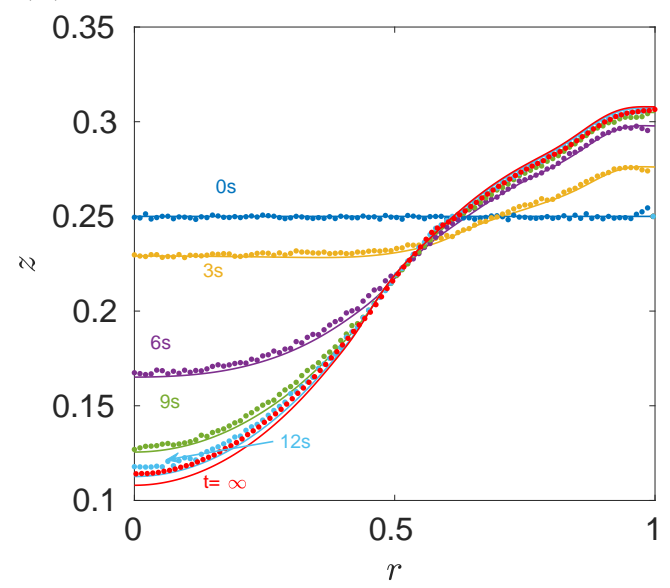

(b)

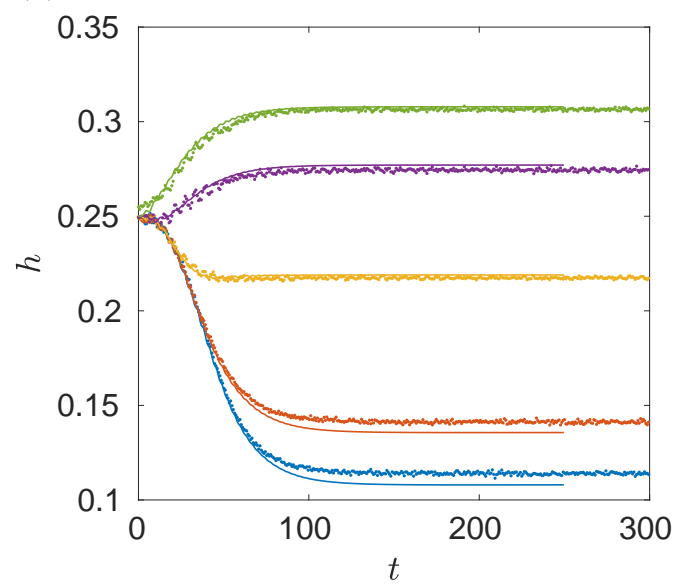

FIG. 5. Case $G=0.25, F r=0.88$ and $R e=3304$ (large setup at $\Omega=7.84 \mathrm{rad} / \mathrm{s}$ and $H=35 \mathrm{~mm}$ ). Comparison between unsteady simulation (solid lines) and experimental measurements (dots). (a) Radial profiles of the liquid surface every 3 seconds from the start of disk rotation, that is $t=0$, 23.52, 47.04, 70.56, 94.08 in dimensionless scale (red for the steady state). (b) Time evolution of the surface height at $r=0,0.25,0.5,0.75$ and 1 . For readability, the experimental signals have been resampled every $0.1 \mathrm{~s}$ instead of using the original temporal resolution of $0.01 \mathrm{~s}$.

The shape of the interface obtained by numerical simulations is compared to measurements in figure 5(a) at regular time intervals during the spin-up and the surface height evolution at several radial positions is shown in Figure 5(b). A very good agreement is generally found between the interface shape obtained numerically and experimentally. The small discrepancies occurring in two regions, close to the axis and in the meniscus, are discussed later (see section III B). 
The azimuthal velocity component $V_{\theta}$ is also measured at several probe locations (see figure $6(\mathrm{a})$ ), chosen in such a way that they remain always in the liquid phase during the spin-up. As the LDV only permits local measurements, each time signal corresponds to a different run of the experiment.
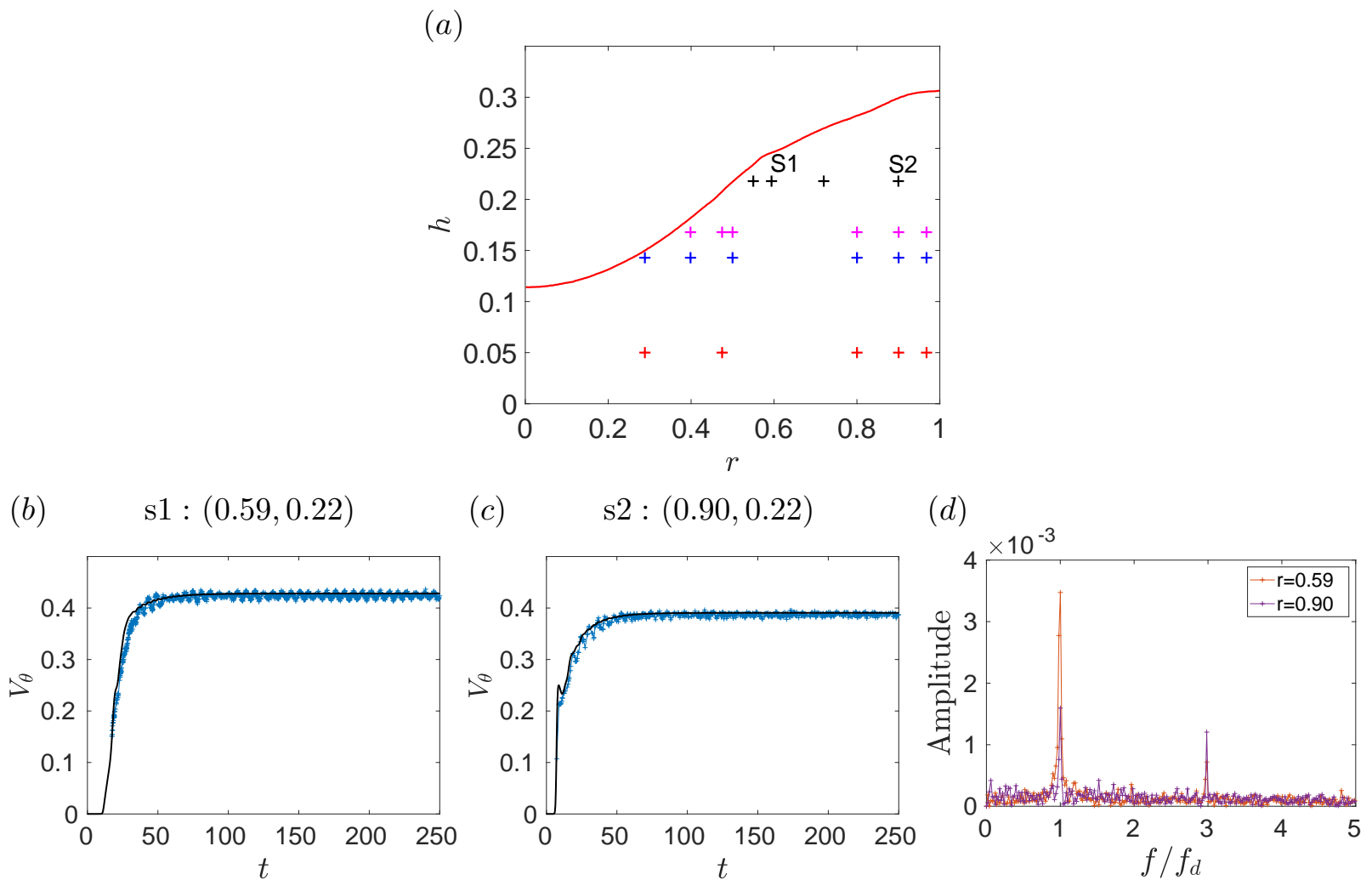

FIG. 6. Same parameters as in fig. 5. (a) Probe locations for the azimuthal velocity measurements. The interface shape measured at steady state is represented by the solid red line. (b,c) Comparison between the numerical (solid) and experimental (symbols) transient evolutions of $V_{\theta}$ at two probe locations $(r, z)$. (d) Amplitude spectrum in the permanent regime for the two time signals s1 and s2 between $t=118$ and $t=432$.

Figure $6(\mathrm{~b}, \mathrm{c})$ displays two examples of such signals along with their numerical counterpart chosen at the same height $z=0.22$. As the radial locations are in the peripheral region, the transient time is roughly 50, fully coherent with an estimation given by the Ekman spin-up time $\tau_{E}=R e^{1 / 2}=57$ (even though the present case is nonlinear, of finite radial extent, with a free surface). One can observe that the velocity starts to increase earlier for the probe located closer to the cylinder wall. This is consistent with the scenario of momentum convection in the meridional plane presented above. Again, a close agreement 
between numerics and experiments is obtained during spin-up. A numerical simulation with the disk started impulsively, i.e. without ramp, has also been performed (not shown): a global time shift of the order of magnitude $\Delta t_{r}$ is then observed but the dynamics are not significantly modified. This is expected since $\Delta t_{r}$ is one order of magnitude smaller than $\tau_{E}$.

\section{B. Permanent regime}

In figures $6(\mathrm{~b}, \mathrm{c})$, it is noticeable that the experimental signal in the permanent regime displays some small amplitude oscillations. The corresponding spectrum is dominated by two peaks at frequencies $f=f_{d}$ and $3 f_{d}$ (see figure $6 \mathrm{~d}$ ). These two frequencies are also present in the measurements of local disk height performed without fluid. We therefore attribute the unsteadiness to the disk wobble and not to flow instability. The two experimental signals averaged over time (here between $t=118$ and $t=432$ ) give values $V_{\theta}=0.424$ and 0.387 , perfectly predicted by the numerical results $V_{\theta}=0.424$ and 0.388 . This close agreement leads us to believe that, apart from this experimental artifact, the system in the permanent regime is very close to an essentially axisymmetric steady state, which is now described.

The radial distribution of the azimuthal velocity $V_{\theta}$ obtained numerically is plotted for 4 different heights in figure 7 , along with the time average of the experimental measurements at each of the 21 probe locations (displayed in figure 6(a)). As the surface is strongly deformed and the velocity can be measured only in the liquid, we have represented the numerical results over a limited range, namely from the radial interface location to the vertical wall. Three regions can be distinguished. In the core region for $r<0.4$, the flow is in solid body rotation at the angular speed of the disk. In a peripheral region for $r>0.5$, the angular velocity $V_{\theta}(r)$ decreases, similarly to the potential region of the Rankine vortex model [16]. In the vertical wall region near $r=1$, a boundary layer develops, associated to a local peak of $V_{\theta}$. As $z$ increases, the intensity of the peak decreases while the thickness of the boundary layer increases $[24,35]$. Except in the boundary layers, the azimuthal velocity is found almost $z$-independent, even though the interface is strongly deformed. Figure 8 enables a better understanding of the flow structure, in particular of the meridional flow bordered by boundary layers, as described in [35] and [24].

In figure 7 , the main discrepancy between numerics and experiments is observed at the 


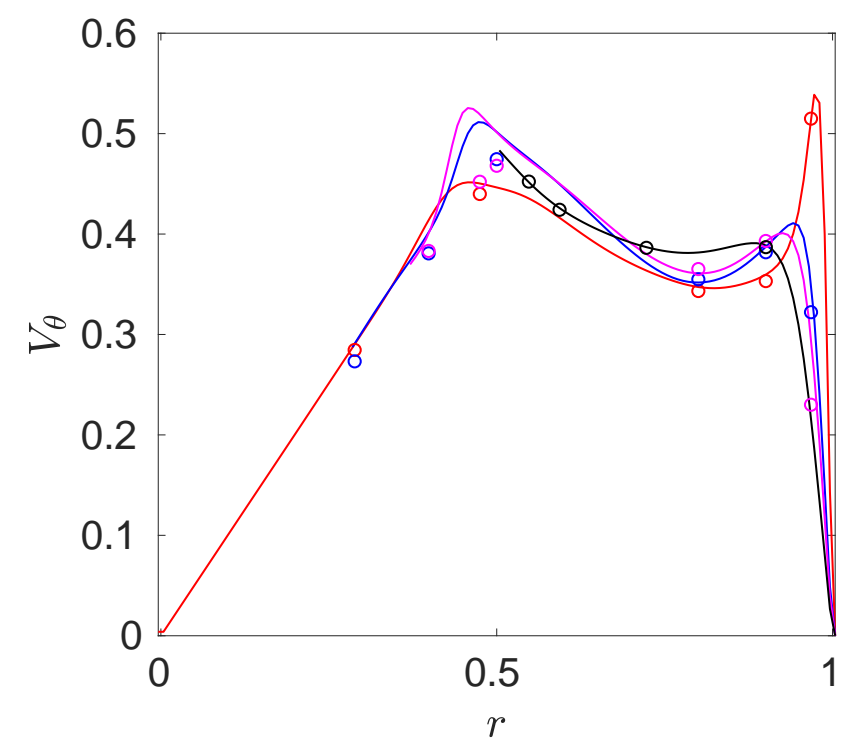

FIG. 7. Same parameters as in fig. 5. Comparison between numerical and experimental steady azimuthal velocities $V_{\theta}(r)$ at four different heights $z=0.05$ (red), 0.14 (blue), 0.17 (magenta) and 0.22 (black). Colors are coherent with those used for the probes in figure 6(a). Solid lines refer to numerical solutions and symbols to measurements.

junction between core and peripheral regions for $0.35<r<0.5$, where an overshoot of the computed azimuthal velocity is present. At these locations, the flow is predicted to rotate faster than the disk, which is not the case in the experimental results (see also [24]). This overshoot is a consequence of angular momentum $r V_{\theta}$ conservation: as fluid elements are convected towards the axis, their azimuthal velocity increases. The existence of the overshoot is thus strongly tied to the intensity of the meridional circulation. We suspect that the radial velocity at the free surface is smaller in the experiment than the numerical prediction, possibly due to surface pollution, so that the overshoot is not observed experimentally. Such radial velocity damping has been observed by Bergmann et al. [4] (their figure 12). The steady state has also been computed using the Newton-based code Rose: figure 8 shows that in the liquid phase the flow fields computed using both codes are almost indistinguishable. Two pieces of information can be deduced: (i) this overshoot is a robust feature of the numerical model, and (ii) the stresses due to air flow on the liquid phase are negligible. Indeed, the air layer is not taken into account in the computations performed using Rose.

The overall comparisons between numerics and experiments are very satisfactory for both the height and velocity profiles. The accuracy of our measurement apparatus allows 
(a)

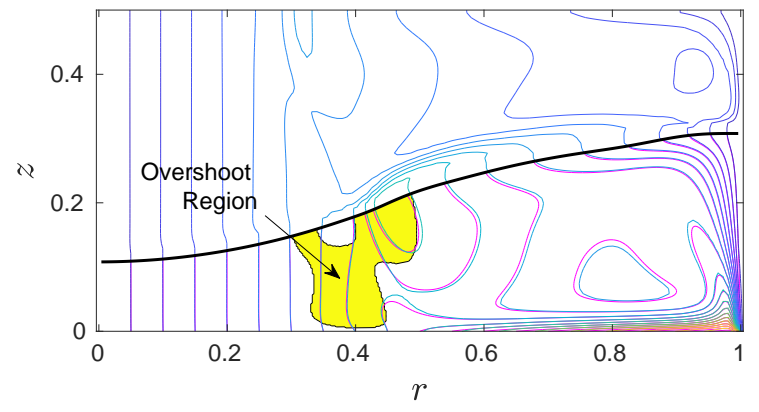

(b)

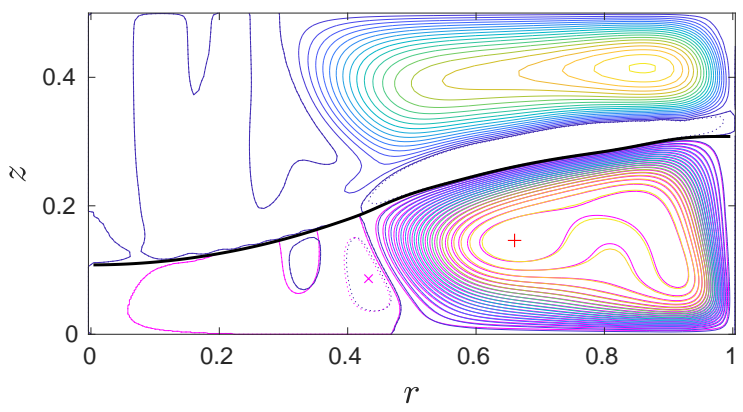

FIG. 8. For the same parameters as in Fig. 5, the steady state is computed using SunfLuIDH (multicolor contours) and Rose (magenta contours). (a) Azimuthal velocity $V_{\theta} ; 21$ equally spaced levels between 0 and 1 . The overshoot region is highlighted (yellow area) in which the azimuthal velocity exceeds the solid body rotation by more than $1 \%\left(V_{\theta}>1.01 r\right)$. The steady interfaces obtained using Rose and SunfluidH (solid black line) are both plotted but are indistinguishable. (b) Streamfunction $\psi$; positive contour values: 21 equispaced levels between 0 and $\psi_{\max }=0.0057$ (the maximum is reached at the same location marked by the ' + ' symbol for both codes); negative contour values: one dashed contour at $\frac{1}{2} \psi_{\text {min }}$ for Sunfluidh (dashed blue) and for Rose (dashed magenta) with $\psi_{\min }=-1.96 \times 10^{-4}$ (the minimum in the liquid phase is reached at almost the same location marked by the magenta ' $X$ ' symbols, negative contours in air phase are not represented). For Rose, the domain $[0,1] \times[0,0.25]$ was used, mapped by a curvilinear grid with $401 \times 101$ points. For Sunfluidh, air parameters read as follows: $\rho_{\text {air }}=1.205 \mathrm{~kg} / \mathrm{m}^{3}, \mu_{\text {air }}=1.82 \cdot 10^{-5} \mathrm{~Pa} \cdot \mathrm{s}$ and the surface tension is $\sigma=65.5 \cdot 10^{-3} \mathrm{~N} / \mathrm{m}$; at the upper boundary $z=0.5$, free slip boundary conditions are imposed.

for investigations of the observed discrepancies. In figure 5 , at $t=0$, the laser line profile sensor captures the meniscus along the vertical wall, where the surface deviates vertically, when converted to dimensional scale, by $1 \mathrm{~mm}$ over a radial distance of about $4 \mathrm{~mm}$. This is of course not captured by numerical simulations for which wettability is not taken into account. Note that as the meniscus physical length scale is not related to the geometrical dimensions of the experiment, the chosen length scale (disk radius) enhances this effect if the disk radius is chosen smaller (see section IV).

Another discrepancy is observed in the vicinity of the axis. Indeed, the surface deviation computed numerically is found larger than the experimental surface deviation. While the 
surface deviation $G-h_{\exp }(r=0)$ reaches $54 \%$ of its initial height, the relative discrepancy

$$
\delta=\frac{h_{\text {exp }}(r=0)-h_{n u m}(r=0)}{G-h_{\text {exp }}(r=0)}
$$

is equal to $4.5 \%$. We relate this difference to the existence, in the numerics, of the region with an overshoot of the azimuthal velocity (shown in figure 8(b)). The overshoot locally induces a larger radial pressure gradient which locally steepens the interface. By mass conservation, this local effect causes a global deviation of the surface: the fluid height in the inner region decreases while it increases in the peripheral one. Artificially damping the velocity overshoot in this small region indeed reduces $\delta$ to $1.2 \%$ (these numerical results are not shown).

\section{Axisymmetry breaking / Occurrence of instabilities}

The rotating bottom disk experiment is known to be prone to instabilities. Investigations reveal two main regimes: (i) a low Froude number regime, for which the control parameter is the Reynolds number, where bulk instabilities (BI) occur [24]; for the present aspect ratio $G=0.25$ such instabilities have been studied by [40]; (ii) a high Reynolds number regime, for which the control parameter is the Froude number, where rotating polygons (RP) prevail $[1,2,5,18]$. Both types of instability break the axisymmetry of the flow since the critical modes have the azimuthal structure $m=2$ or 3 .

In the present study, we focus on flow regimes that have neither a low Froude number, nor a high Reynolds number, so that either type of instability may occur. We can distinguish unsteadiness due to BI, RP or disk wobbling in the following way. Whenever BI or RP are triggered, a rotating pattern is observed on the free surface and the azimuthal wavenumber $m$ can be easily found from light reflections even for tiny deflections of the free surface. For disk wobbling, no pattern is visible. The dominant frequency $f$ of the azimuthal velocity LDV signal associated to the observed mode $m$ then yields its angular speed $\varpi=2 \pi f / m$. For the present aspect ratio, $\varpi$ is close to $2 \Omega / 3$ for $\mathrm{BI}[24]$ while for RP it is close to $\Omega / 3$ $[6]$.

In section IIIB, no instability was found and oscillations were shown to be related to mechanical wobbling (see fig. 6d). Running the experiment with two setups (large and small) at the exact same Froude number allows us to examine the effect of instabilities on the temporal mean surface height. In the small setup, the disk angular speed is set at 
$\Omega=11.12 \mathrm{rad} / \mathrm{s}$, the temperature is $T=24.1^{\circ} \mathrm{C}$. G8W2 is replaced by a mixture of $68.86 \%$ glycerol and $31.14 \%$ water, which has a dynamic viscosity of 19 times that of water at this temperature. This leads to parameter values $F r=0.88$ and $R e=3732$. Note that this Reynolds number is slightly above the value $R e=3304$ studied in section III B in the large setup. Parameter values for both experiments are summarized in table II.

\begin{tabular}{ccccc}
\hline setup & symbol & $F r$ & $R e$ & $W e$ \\
\hline large & $\mathrm{L}$ & 0.88 & 3304 & 3151 \\
small & $\mathrm{S}$ & 0.88 & 3732 & 748 \\
\hline
\end{tabular}

TABLE II. Comparison between flow parameters used for the two experiments at the same Froude number. In both situations, the Weber number is large enough so that the surface tension has little influence on the shape of the interface, except in the meniscus.

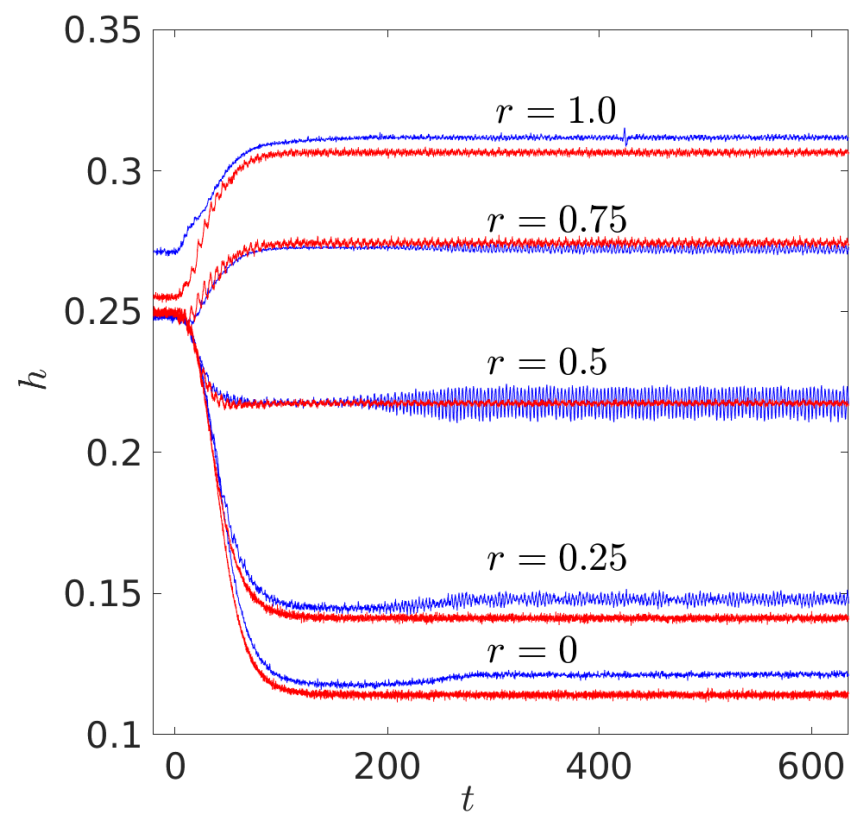

FIG. 9. Comparison of surface evolution at several probes between the case 'L' (red lines) and the case 'S' (blue lines).

The surface deformation measured during each of the two spin-up experiments are compared at several radial locations, as shown in figure 9 . The probe located at $r=0.5$ indicates that, after the completion of spin-up at $t \approx 150$, oscillations increase progressively in the 
small setup only, and saturate at $t \approx 280$. This scenario excludes the possibility that these oscillations are due to disk wobbling. From a spectrum analysis and surface observations, it can be deduced that the azimuthal phase velocity of the pattern is approximately $0.6 \Omega$, which characterizes BI [24]. This instability is indeed present at the junction between the core and the peripheral region, hence around $r=0.5$ where the maximum amplitude is observed [13]. Moreover, this occurrence of instability is coherent with the increase in Reynolds number from the large to the small setup.

The comparison between both experimental signals for $200<t<280$ at $r=0$ and 0.25 shows that the instability induces a distortion of the surface mean height, contrary to disk wobbling. For example, $h_{\text {exp }}(r=0)$ for the small setup increases by about 0.0035 during instability growth, and the discrepancy between $h_{\exp }(r=0)$ in the two setups finally represent $5.3 \%$ of the surface deviation at the axis.

At $r=1$, meniscus effects are significant, and we do not expect to have an agreement in this region (see upper curves in fig. 9). Along the vertical wall at $t=0$, the surface rises by $0.8 \mathrm{~mm}$ (large setup) and $1.6 \mathrm{~mm}$ (small setup), or using dimensionless variables $\Delta h=5.7 \cdot 10^{-3}$ (large setup) and $\Delta h=2.3 \cdot 10^{-2}$ (small setup). This discrepancy weakens throughout the spin-up.

Using the same parameters as for the two experiments (table II), unsteady simulations of the spin-up have been performed in the axisymmetric framework which filters out BI and RP. In figure 10a, surface height distributions obtained numerically in the permanent regime are plotted, together with the temporally averaged experimental distributions for $400<t<600$. The standard deviation of the experimental signals is also represented on the curves using vertical bars. The numerical solutions are very close to each other, which illustrates the weak influence of $W e$ and $R e$ numbers. Most discrepancies between numerics and experiments are observed in the core region. As already mentioned, the experimental height in the large setup rises $(\delta=4.5 \%)$ mainly due to a different azimuthal velocity distribution in the region matching core and periphery (overshoot). This difference is increased up to $\delta=11.3 \%$ in the small setup due to the occurrence of BI.

In order to further analyse the surface geometry in the permanent regime, a rescaled height deformation is introduced:

$$
\widetilde{h}(r)=\frac{\bar{h}(r)-G}{F r}
$$




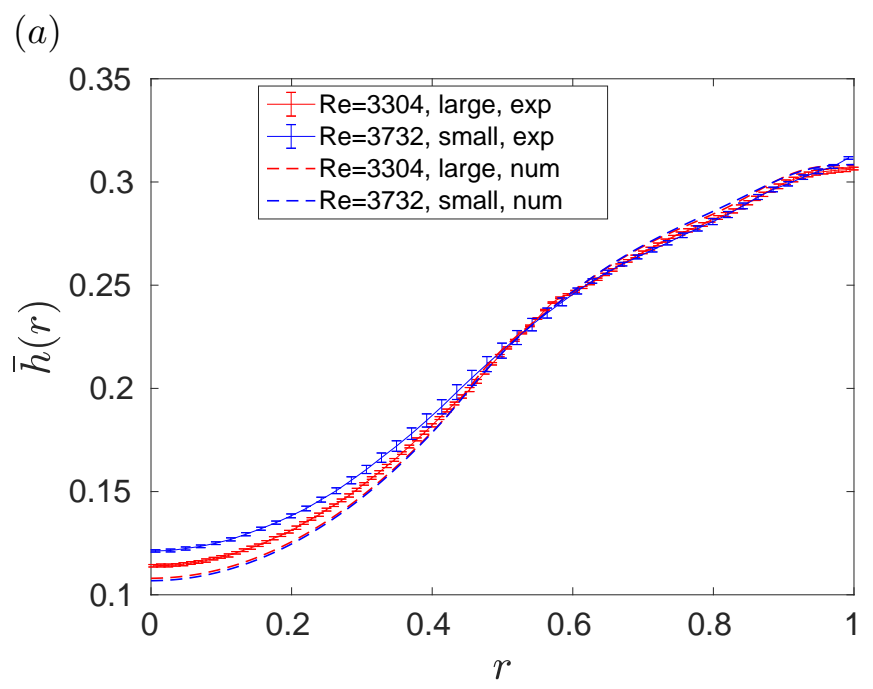

$(b)$

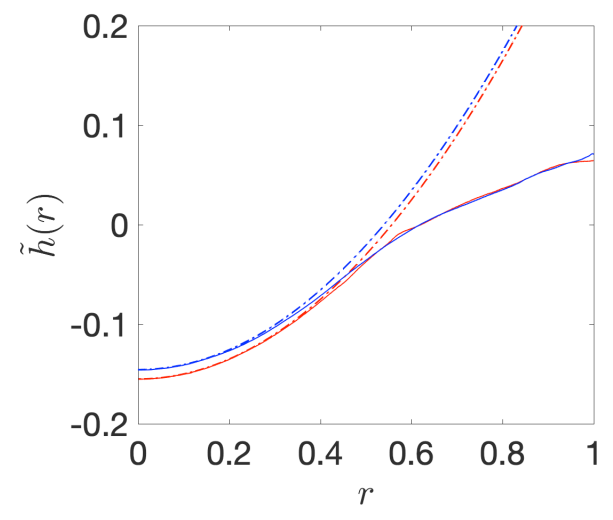

(c)

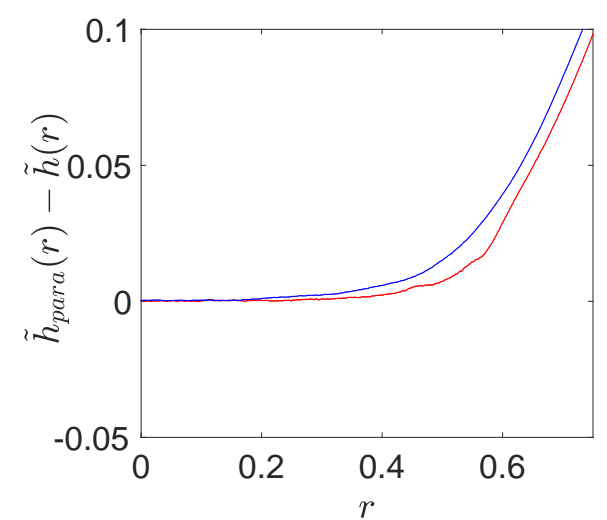

FIG. 10. Comparison of radial height distributions for large (red curves) and small (blue curves) setup for the parameter values of table II. (a) Temporally averaged surface height for $400<t<$ 600 in experiments (solid lines) and numerical results (dashed lines). (b) Rescaled function $\widetilde{h}(r)$ for experiments (solid lines), together with the parabolae $\widetilde{h}_{\text {para }}$ (eq. 6) where $\widetilde{h}_{0}=-0.155$ and $\widetilde{h}_{0}=-0.1456$ are taken from their experimental values in the large and small setups respectively. (c) Difference $\widetilde{h}_{\text {para }}(r)-\widetilde{h}(r)$.

For Newton's bucket, this function would be a parabola $\widetilde{h}_{N}=\frac{1}{2} r^{2}-\frac{1}{4}$. For the disk-driven rotating flow, only the core region is in solid body rotation with the bottom disk, so that we expect the surface height in this region to behave as:

$$
\widetilde{h}_{\text {para }}(r)=\frac{1}{2} r^{2}+\widetilde{h}_{0}
$$

In equation $(6), \widetilde{h}_{0} \equiv \widetilde{h}(r=0)$ depends on the velocity distribution of the peripheral region, 
and is taken from its experimental value. Figure $10 \mathrm{~b}$ displays the two functions $\widetilde{h}$ and $\widetilde{h}_{\text {para }}$ for both experiments. In the core region, it is found the curves $\widetilde{h}(r)$ are superimposed to the curves $\widetilde{h}_{\text {para }}(r)$, which indicates that the fluid indeed rotates at the same velocity as the disk. The radius at which this agreement no longer holds is close to 0.5 for the large setup, and slightly smaller for the small setup where BI is present. Plotting the distribution of $\widetilde{h}_{\text {para }}(r)-\widetilde{h}(r)$ (figure 10c) shows this point.

In short, bulk instabilities when present only moderately affect the shape of the surface, which is slightly lifted up in the core region and remains parabolic. 


\section{FROM WETTED TO DEWETTED}

Using the small setup filled with the G8W2 mixture, we now characterize the surface deformation for cases with the rotating speed $\Omega$ gradually increased. We followed the protocol presented in figure 3 to conduct spin-up experiments (with $\Delta t_{r}=1 \mathrm{~s}$ and $\Delta t_{p}=60 \mathrm{~s}$ ) and focus on time-averaged surface heights in the permanent regime. The liquid temperature is around $T=28^{\circ} \mathrm{C}$. Parameter values of the different cases are reported in table III.

\begin{tabular}{cccc}
\hline exp. & $\Omega(\mathrm{rad} / \mathrm{s})$ & $F r$ & $R e$ \\
\hline 1 & 7.85 & 0.44 & 1208 \\
2 & 11.66 & 0.97 & 1794 \\
3 & 13.11 & 1.23 & 2017 \\
4 & 14.57 & 1.51 & 2242 \\
5 & 15.54 & 1.72 & 2391 \\
6 & 19.00 & 2.58 & 2924 \\
\hline
\end{tabular}

TABLE III. Parameters used to characterize the surface deformation as the rotating speed $\Omega$ is increased.

Figure 11(a) shows the time-averaged surface heights obtained in the permanent regime, from measurements and numerical simulations. For the physical parameters listed in table III, numerical curves have been obtained with SUNFLUIDH code using the same protocol and numerical parameters as in section III. As the Froude number increases, the fluid is being expelled toward the periphery by the centrifugal acceleration. Above a critical value, the fluid height vanishes at the center of the disk, which yields dewetted regimes. A close match between numerics and experiments is obtained for cases 1 and 6 whereas for cases 2-5, some discrepancies are observed. As explained in section III C, these discrepancies come from the persistence of an overshoot of the azimuthal velocity in the numerics and also the development of $\mathrm{BI}$ in the experiments. On closer inspection of the temporal evolution of surface height in the permanent regime (not shown), the maximum oscillation amplitude due to BI increases with the Reynolds number from $0.15 \mathrm{~mm}$ for case 1 , up to $0.8 \mathrm{~mm}$ for case 3, and decreases thereafter. The discrepancy between experimental and numerical time-averaged surface height evolves in the same way. It reaches a maximum for case 3, where the relative error $\delta=10.5 \%$. For case 6 where the center of the disk is dewetted, BI is 
(a)

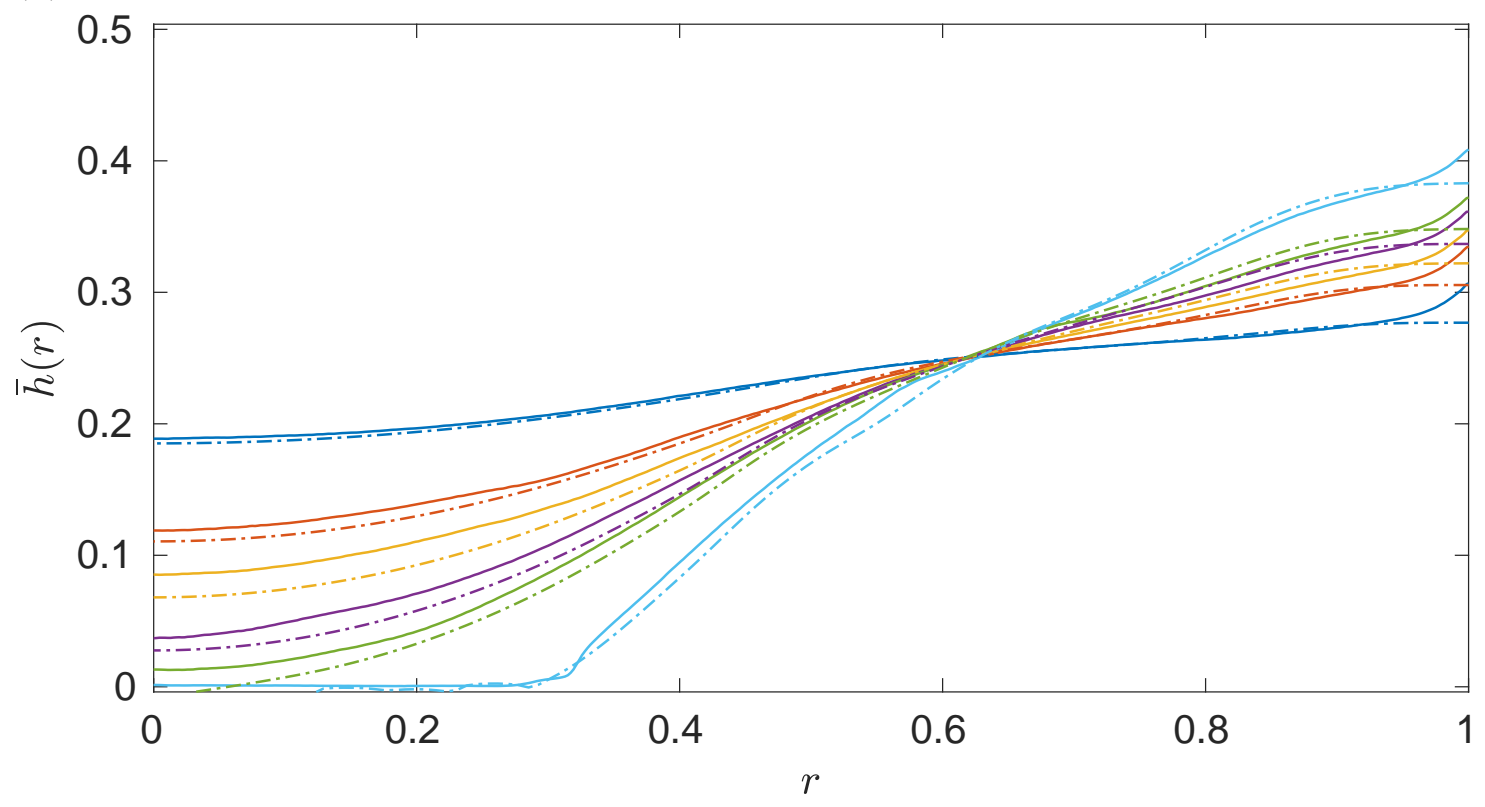

(b)

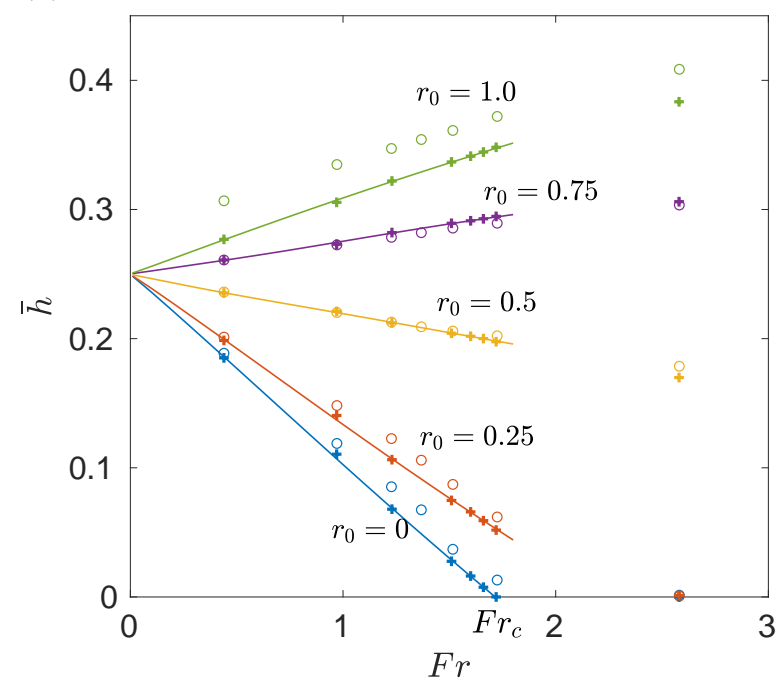

$(c)$

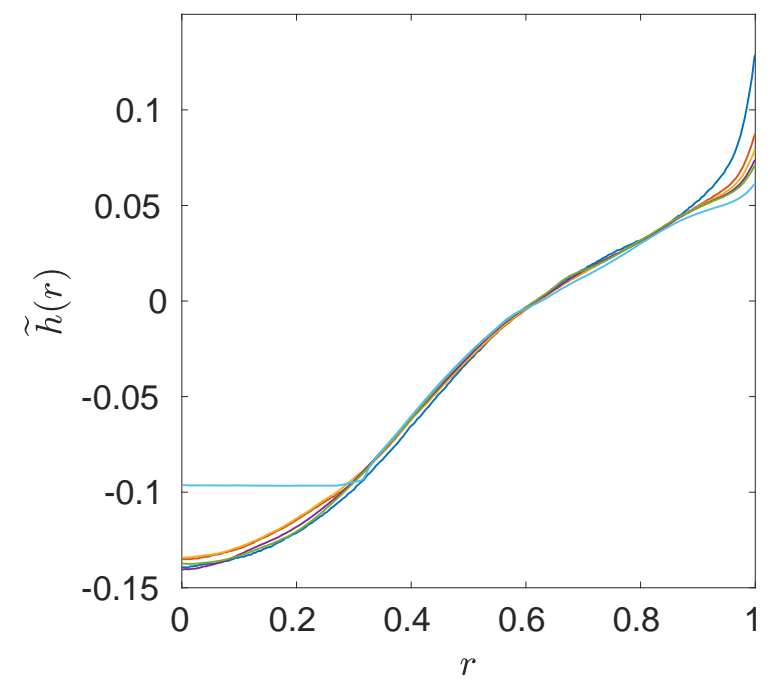

FIG. 11. (a) Time-averaged surface height distributions for cases 1-6 in table III. Measurements (solid lines) and numerical results (dashed lines). From top to bottom at $r=0$ : $F r=0.44,0.97,1.23,1.51,1.72,2.58$. (b) Surface height at $r=0,0.25,0.5,0.75,1.0$ as a function of Froude number. ○: measurements; +: SunfluidH results in the permanent regime; - Rose results (up to dewetting). (c) Rescaled surface height distributions $\widetilde{h}(r)$ as defined in Eqn (5) for the experimental cases of (a). All experiments are performed on the small setup with G8W2 as working fluid at $T \approx 28^{\circ} \mathrm{C}$ with a layer of initial depth $17.5 \mathrm{~mm}$. 
damped, the spectrum is now dominated by the wobbling frequency and a close agreement is then recovered.

Figure 11 (b) shows the surface height at several radial locations as a function of the Froude number. Experimental values along with simulations performed with SuNFLUIDH and Rose (on a mesh $N_{r} \times N z=201 \times 51$ ) are represented. The results obtained from the different codes agree quite well. Figure 11 (b) exhibits a linear behavior of the surface height with the Froude number. The threshold $F r_{c}$ above which dewetting occurs is found numerically around 1.72. Note that using the linear dependency on $\mathrm{Fr}$, one could have predicted a good approximation by extrapolating the values $h(0)=0.25$ at $F r=0$ and $h(0)=0.1851$ at $F r=0.44$, which would yield $F r_{c}=1.70$.

All other physical quantities being fixed, $R e$ and $F r$ vary (respectively linearly and quadratically) with the only control parameter $\Omega$. However, the Froude number is the key parameter when surface deformation is addressed. Indeed, when the surface height distributions are rescaled using $\mathrm{Fr}$ as defined in equation (5), all curves collapse on a master curve, as seen in figure 11(c). This property is all the more striking as it is valid over a large range of Froude numbers, including case 6 for which the disk is dewetted, except of course in the dewetted region $r<0.3$. This rescaling also emphasizes the minor influence of the Reynolds number, at least in the parameter range listed in table III. As expected, this $\mathrm{Fr}$ similarity does not hold in the meniscus region $r>0.9$ as the capillary length remains roughly constant for all experiments, which creates an artificial distortion as Froude number is varied. 


\section{SPIN-UP AND PERMANENT REGIMES WITH DISK DEWETTING}

In the following, we focus on the dewetted disk regime, which is obtained for $F r>F r_{c} \approx$ 1.72. Centrifugal acceleration now exceeds gravity, which strongly leans the constant pressure interface. The small experimental setup is handy to reach such large Froude numbers while maintaining moderate Reynolds numbers. This results in permanent flows without RP. The tank is filled with a G8W2 layer of initial depth $17.5 \mathrm{~mm}$. The disk is uniformly accelerated from rest during $\Delta t_{r}=1 \mathrm{~s}$ to reach the plateau speed. On the numerical side, we use the same procedure as for the wetted case of section III.

\section{A. Spin-up at $F r=2.58$}

The plateau angular speed is $\Omega=19.00 \mathrm{rad} / \mathrm{s}$ and the temperature is $27.5^{\circ} \mathrm{C}$, corresponding to $F r=2.58$ and $R e=2847$. The shape of the interface obtained by experimental and numerical approaches is plotted in figure 12(a) at regular time intervals during the spin-up and the surface height evolution at several radial positions is shown in figure 12(b).

$(a)$

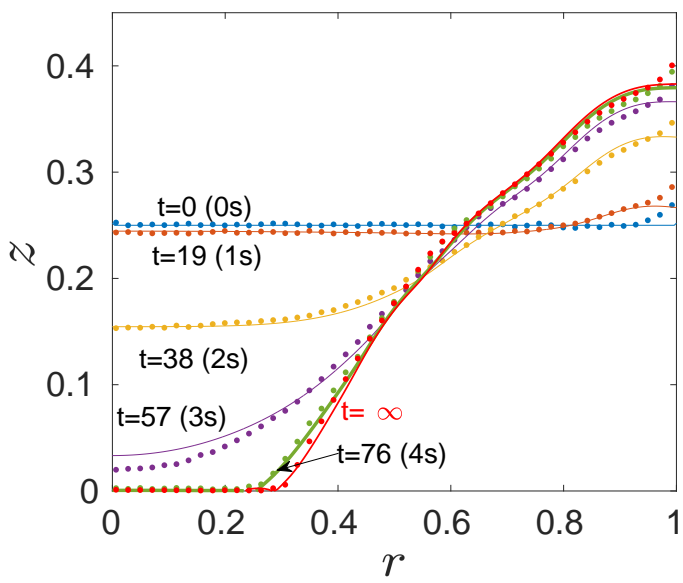

(b)

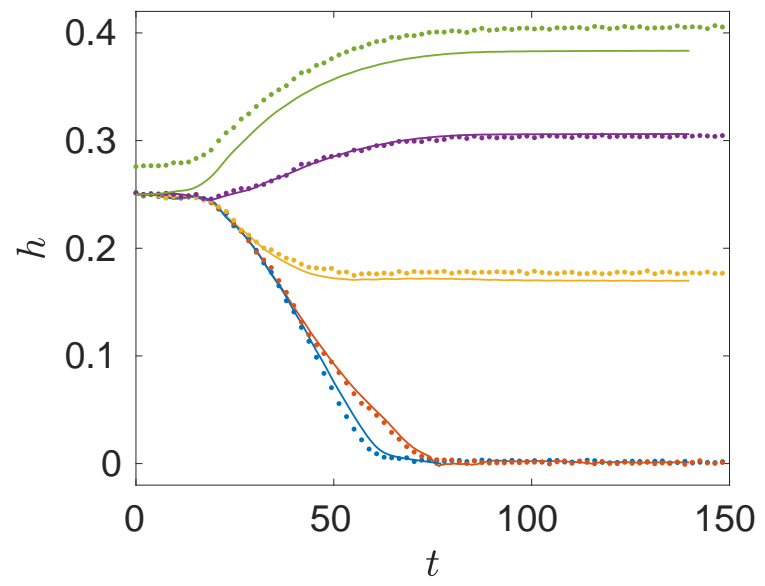

FIG. 12. Case $G=0.25, F r=2.58$ and $R e=2847$ (small setup at $\Omega=19 \mathrm{rad} / \mathrm{s}$ and $H=$ $17.5 \mathrm{~mm}$ ). Comparison between unsteady simulation (solid lines) and experimental measurements (dots). (a) Radial profiles of the liquid surface every second from the start of disk rotation, that is $t=0,19,38,57,76$ in dimensionless scale (red for the steady state). (b) Time evolution of the surface height at $r=0,0.25,0.5,0.75$ and 1 . For readability, the experimental signals have been resampled every $0.1 \mathrm{~s}$ temporally and $1.5 \mathrm{~mm}$ spatially. 
Except in the meniscus region, a very good agreement is generally found. The discrepancy observed in figure 12(a) at the axis for $t=57$ (dimensional time $3 \mathrm{~s}$ ) is about $\delta=4.8 \%$ but it should be put into perspective with the rapid evolution of the system as the surface becomes closer to the bottom disk (see lower curve Fig. 12(b)). This corresponds to a small lag of approximately $\delta t=2$ (dimensional $0.1 \mathrm{~s}$ ) between experiment and numerics. Dewetting occurs roughly at $t=63$ (dimensional time $3.3 \mathrm{~s}$ ) and is seen to be perfectly captured by the numerics. A movie is provided as supplementary material[41]. In a simulation with an impulsive start of the disk $\left(\Delta t_{r}=0\right)$, dewetting occurs roughly $1 \mathrm{~s}$ earlier which shows that the ramp does not affect much the dynamics of the interface.

Snapshots of the flow structure are depicted in figure 13. The early evolution is similar to the wetted case: for instance, figure 13a,b and figure 4e,f are comparable. Yet, as time increases, the fluid height decreases in the central region and reaches the boundary-layer thickness near $t=57$ (Fig. 13c,d). This has two main consequences in this region: (i) the axial gradient of the azimuthal velocity becomes weak, which inhibits Ekman pumping; (ii) the solid body rotation induces a curvature of the interface via centrifugal acceleration. During the process, the fluid is flushed towards the periphery at a velocity comparable to that of the interface, which is here one order of magnitude smaller than the typical velocities in the meridional plane. The dewetted region then grows rapidly in size without much structural change of the peripheral region (Fig. 13e,f) and stabilizes at a radius $r_{\mathrm{w}}=0.29$ (see for instance Fig. 16).

We now investigate the spin-up process inside the liquid layer with LDV measurements of the time-dependent azimuthal velocity. As such measurements are performed in a sequential manner, temperature variations could not be avoided, but have been recorded between $22.4^{\circ} \mathrm{C}<T<27.9^{\circ} \mathrm{C}$. As a consequence, the Reynolds number may vary between $R e=2140$ and 2910. As long as instabilities are not triggered, this seemingly large range of Reynolds number does not affect these results significantly (see section VB). Simulations are run at fixed temperature $T=25^{\circ} \mathrm{C}$ corresponding to $R e=2480$. Among all the probe locations investigated, figure 14a displays four locations where comparisons between measured and simulated azimuthal velocity are presented (figure 14b-e). As probes s2, s3 and s4 are initially located $0.7 \mathrm{~mm}$ under the horizontal surface, the measurements are somewhat sparse in time for $t<25$ due to particle sedimentation. They become well time-resolved as fresh particles are brought there by the meridional flow. As probes s2 and s3 remain in the 
(a)

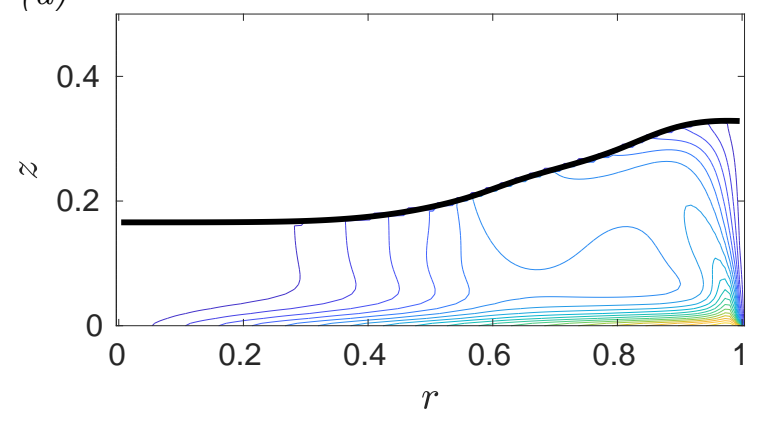

(c)

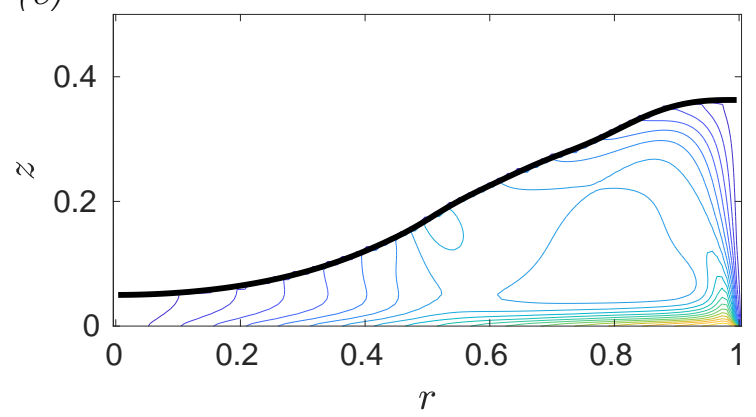

(e)

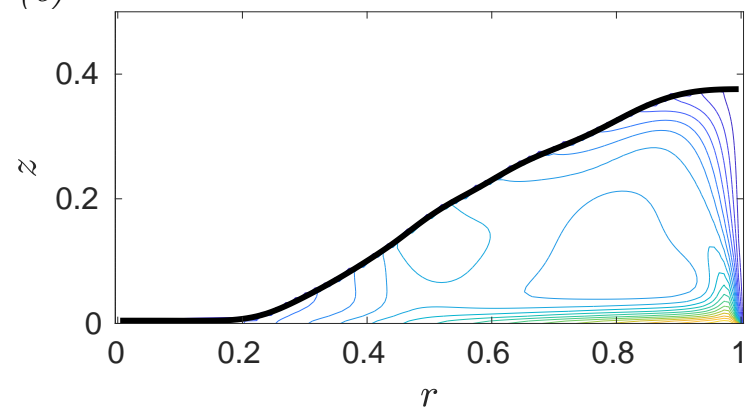

(b)

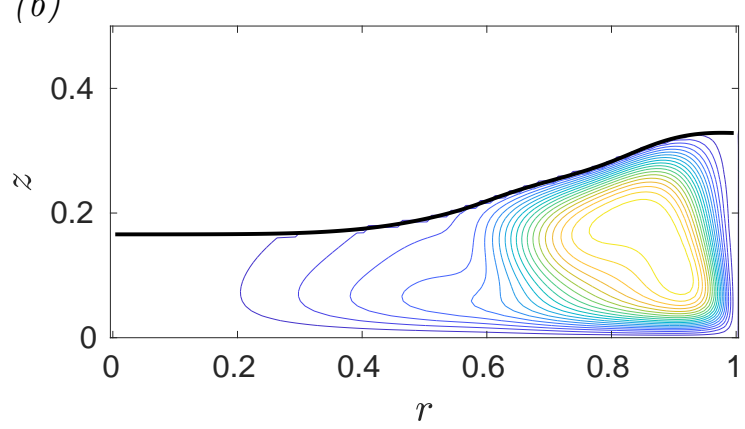

(d)

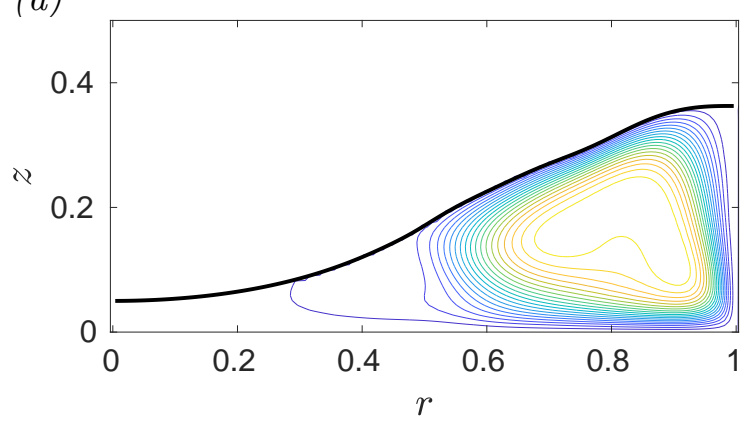

(f)

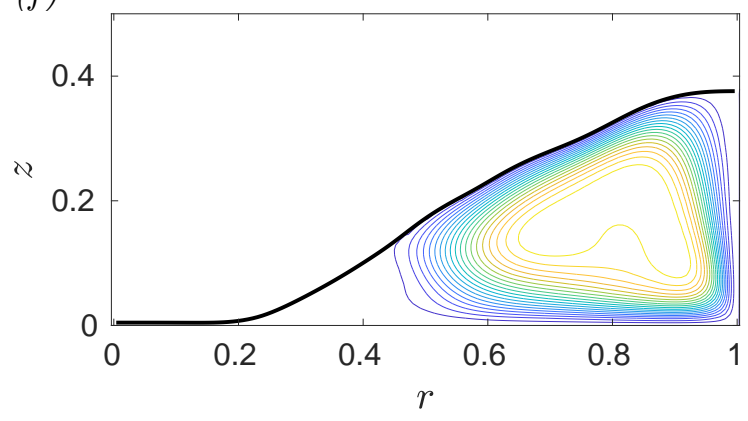

FIG. 13. Snapshots of the computed flow field in the liquid phase from top to bottom at $t=38$, 57 and 72 (dimensional time $2 \mathrm{~s}, 3 \mathrm{~s}$ and $3.8 \mathrm{~s}$ ) corresponding to the spin-up of the experiment for the case $G=0.25, F r=2.58$ and $R e=2847:$ small setup at $\Omega=19.0 \mathrm{rad} / \mathrm{s}$ and $H=17.5 \mathrm{~mm}$. (a,c,e): iso-contours of $V_{\theta}$ with 21 equispaced contours between 0 and 1. (b,d,f): Streamlines of the meridional circulation consisting of iso-contours of $\psi$ with 21 equispaced levels between 0 and $\psi_{\max }$ (in the liquid phase) with $\psi_{\max }=0.0060,0.0063,0.0065$. The black lines in all the graphs represent the numerical air-liquid interface.

vicinity of the deformed surface throughout the spin-up process, a perfect agreement between numerics and experiment is difficult to reach, probably due to unmodelled surface effects. The agreement is better for probe s4 and excellent for s1 which is located in the bulk. 


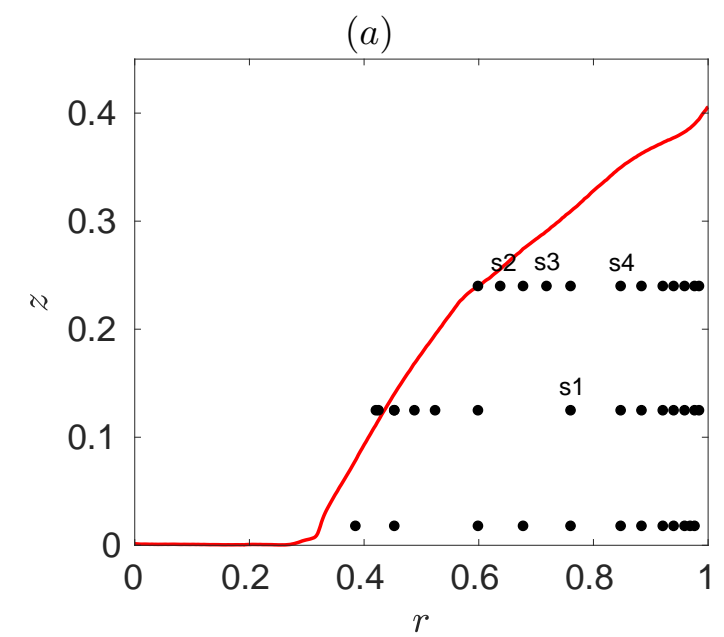

(b) $\mathrm{s} 1:(0.76,0.125)$

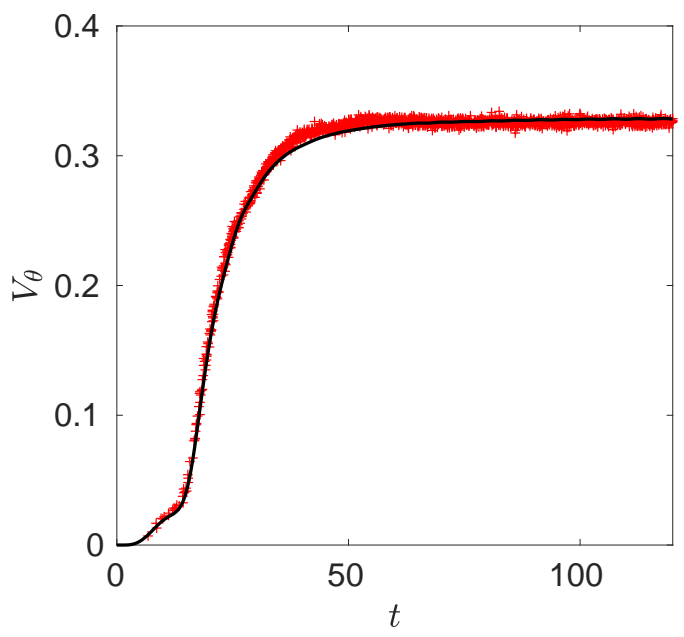

(c) s2: $(0.6,0.24)$

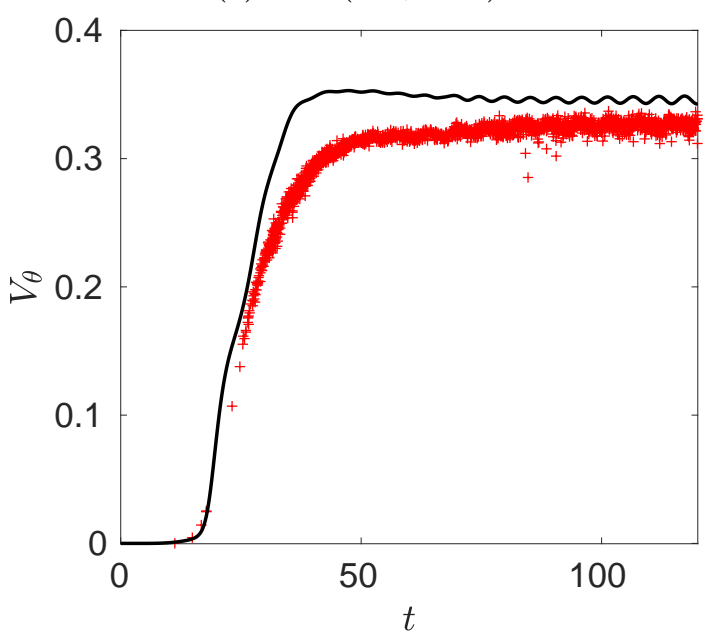

(d) s3 : $(0.72,0.24)$

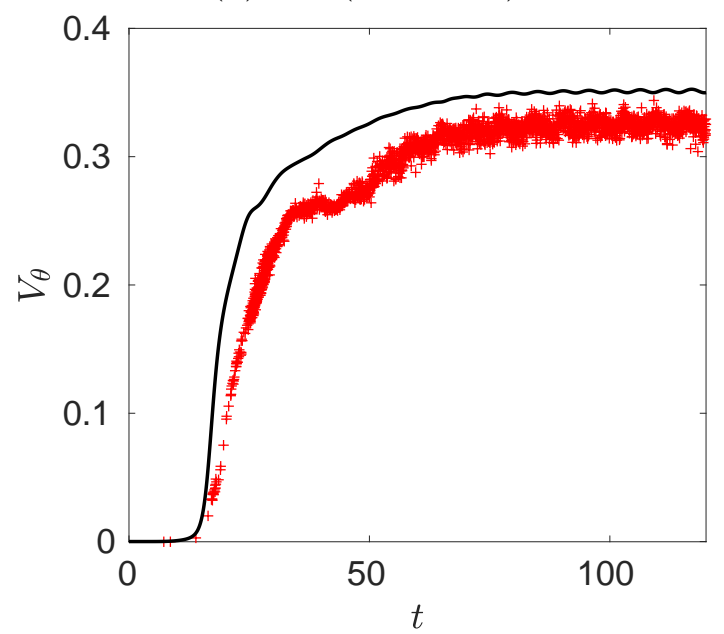

(e) $\mathrm{s} 4:(0.85,0.24)$

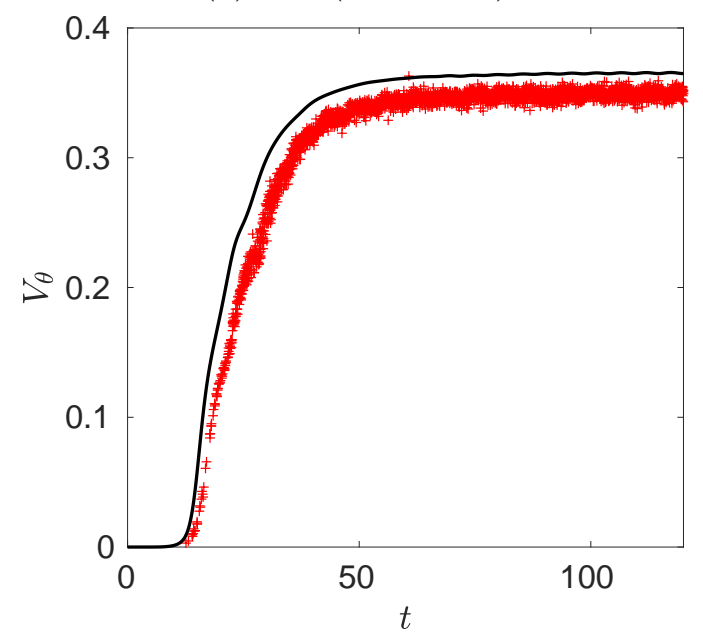

FIG. 14. (a): Probe locations for LDV measurements for the case $G=0.25, F r=2.58$. The measured surface profile in the permanent regime is also plotted. (b)-(e): Temporal evolution of $V_{\theta}$ at four probe locations $(r, z)$, obtained by LDV measurements (red + symbols) and simulation at $R e=2480$ (black solid lines). 


\section{B. Permanent regime}

Measurements of the time-averaged azimuthal velocity profiles in the permanent regime are shown in figure 15 . Numerical results at $R e=2480$ converge to a steady state and are also presented in the same figure. A spectral analysis of the experimental signals reveals that the time dependence is dominated by a weak disk-wobbling effect. Fig. 15a depicts the radial profile near the disk at $z=0.018$. Except near the fixed lateral wall, $V_{\theta}$ increases with $r$. As the disk is dewetted, fluid in solid body rotation is only observed in a narrow range extending from the free surface at $r=0.32$ up to $r \approx 0.45$. An excellent agreement between the numerical and experimental velocities is obtained despite the strong axial gradient of azimuthal velocity within the bottom disk layer. Such good agreement also holds for the velocities measured within the vertical layers along the fixed wall, as can be seen in figures 15b,c. Overall, the only discrepancy observed is again in the overshoot region at $r \approx 0.45$ for $z=0.125$ (see also overshoot region in Fig. 16). Note that in order to evaluate the influence of varying temperature, two other Reynolds numbers $R e=2140$ and 2910 are also plotted in figure 15. This shows a very limited effect of the Reynolds number on the flow field.

(a) $z=0.018$

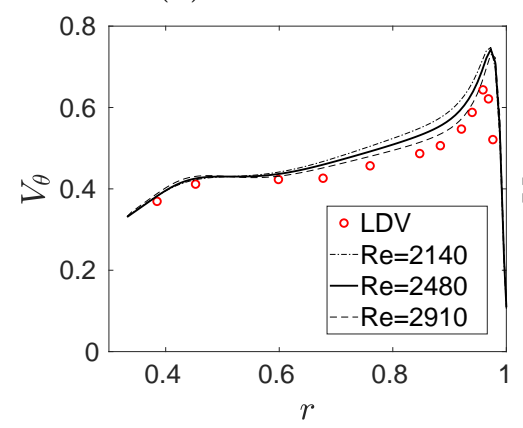

(b) $z=0.125$

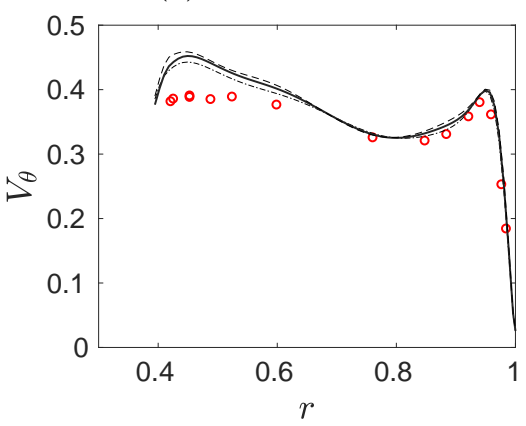

(c) $z=0.24$

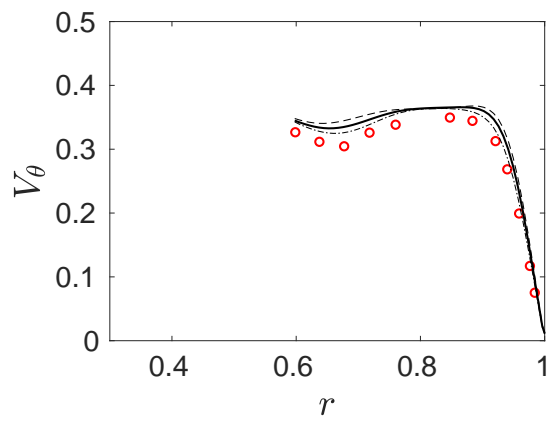

FIG. 15. Radial profile of $V_{\theta}$ at the three measured heights for the case $G=0.25, F r=2.58$. Circles indicate measurements and solid lines numerical results at $R e=2480$. Steady velocity profiles at $R e=2140$ and 2910 obtained numerically are also plotted.

The flow structure obtained from numerical simulations is shown in figure 16. Isocontours of $V_{\theta}$ in the liquid phase confirm that at $F r=2.58$, the solid body rotation is confined in a narrow annular region at $r<0.4$ (see Fig. 16a). Moreover, the meridional circulation is strongly constrained by the gas-liquid interface, so that the surface layer is directly connected 
(a)

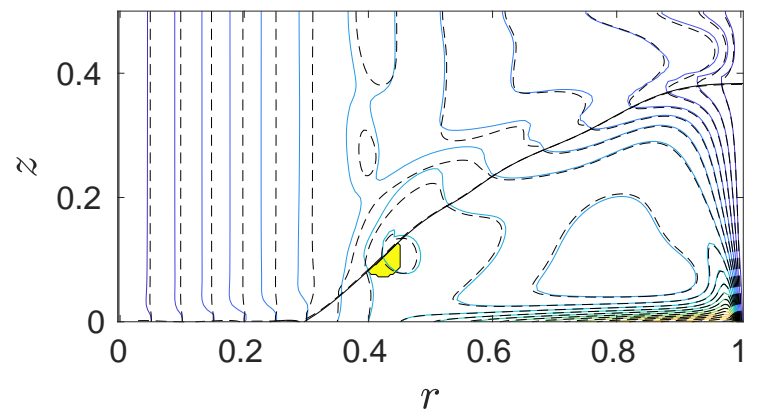

(b)

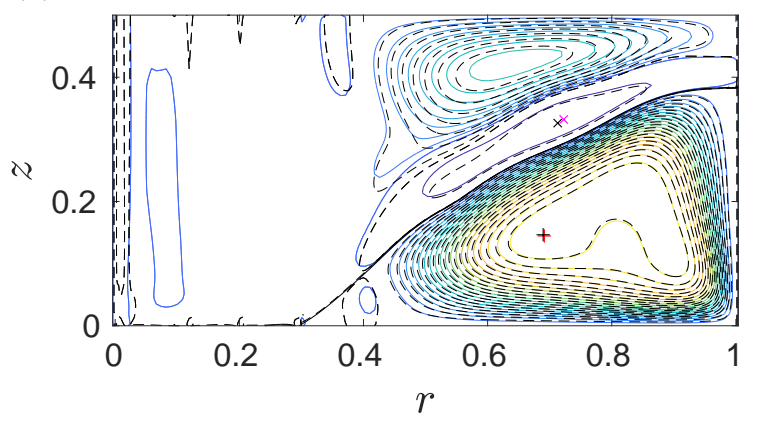

FIG. 16. For the same parameters as in figure 12, the velocity field is computed by SunfLUIDH with $128 \times 64$ cells (multicolor) and $256 \times 128$ cells (dashed black lines). The black lines in the two graphs represent the numerical air-liquid interface (indistinguishable for both mesh). (a) Isocontours of $V_{\theta}$, with 21 equispaced contours between 0 and 1 . The overshoot region (yellow area) is defined as in Fig. 8. (b) Streamlines of the meridional circulation consisting of iso-contours of $\psi$ with 21 equispaced levels between 0 and $\psi_{\max }=0.0065$ (red ' + ') (the same maximum is found for both mesh) and one dashed contour at $\frac{1}{2} \psi_{\min }$. The minimum, red and black ' $\times$ ' located in the air phase, differs significantly as $\psi_{\min }=-0.0034$ and $\psi_{\min }=-0.0027$ for $128 \times 64$ and $256 \times 128$ grid respectively. For SunfluidH, air parameters and boundary conditions are as in Fig. 8.

to the bottom disk layer (Fig. 16b). The azimuthal velocity profile measured at $z=0.24$ can now be better understood. At this constant height, the LDV measurement line crosses both the surface layer from $r=0.6$ to 0.75 and the vertical side wall layer from $r=0.9$ to 1 , leading to the profile presented in figure 15c.

In figure 16, isocontours from SUNFLUIDH simulations using two different meshes $128 \times 64$ and $256 \times 128$ are superimposed. The coarser mesh is found to be sufficient to obtain converged results regarding the interface location and the velocity field in the liquid phase. Reaching convergence for the velocity in the gas phase is clearly more demanding. Nevertheless, this comparison illustrates again that the gas phase has no influence on the liquid phase.

We finally focus on the distribution of the time-averaged azimuthal velocity when the Froude number is varied. An idealized model for the free surface shape and the azimuthal velocity profile has been proposed by [15] for large surface deformation, then extended by [16] to small and moderate deformations. Three possible cases were identified: wet-composite, dry-composite and dry-potential, respectively modelling a wetted case, a dewetted case with 
(a)

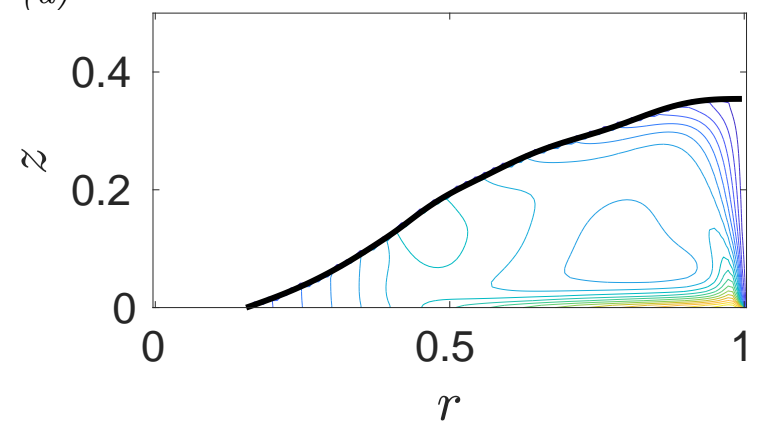

(b)

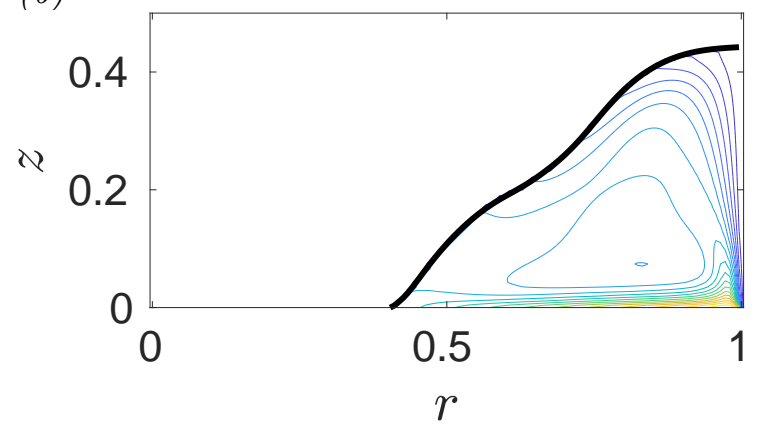

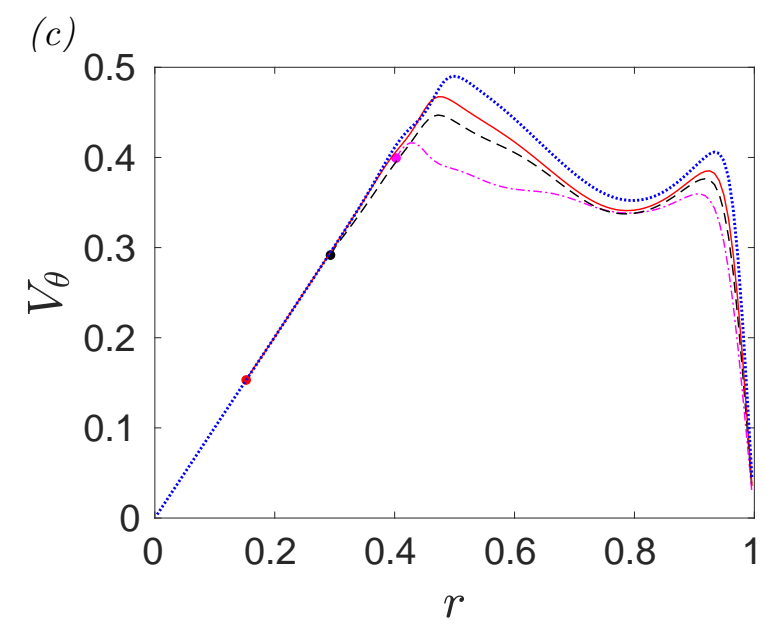

FIG. 17. Effect of the Froude number on the azimuthal velocity in the permanent regime at Reynolds number $R e=2847$. (a,b) Iso-contours of $V_{\theta}$, with 21 equispaced contours between 0 and 1, together with the liquid-gas interface (bold line). The Froude number is (a) $F r=1.8$ and (b) $F r=5$. (c) Radial distributions of the azimuthal velocity $V_{\theta}$ at $z=h(r) / 2$ for the wetted case $F r=0.88$ (blue dotted) and three dewetted cases $F r=1.8$ (red solid), 2.58 (black dashed) and 5 (magenta dot-dashed). The solid symbols indicate the positions $r=r_{\mathrm{w}}$ of the triple line at the disk, respectively equal to $0.15,0.29$ and 0.4 for the three dewetted cases.

solid body rotation, and a dewetted case without solid body rotation. Illustrations of such cases in the viscous context are respectively obtained at $F r=0.88$ (figure 8), $F r=1.8$ (figure 17a) and $F r=5$ (figure 17b). The idealized model supposes a $z$-independent profile for the velocity, which would be expected here only at very high Reynolds numbers as boundary layers become very thin. In the present study, the Reynolds number is moderate, but it is possible to extract azimuthal velocity profiles in qualitative agreement with the above-mentioned models along a line located at mid-height between bottom and free surface (figure 17c). Indeed, the choice of mid-height allows one to avoid as much as possible surface 
and bottom layers; this is pertinent up to $r=0.8$ where the effects of the side layer are felt. 


\section{CONCLUSION}

The goal of this study is to accurately describe the base flow leading to rotating polygonal patterns, yet remaining under but close to the instability threshold. This flow is characterized by moderate to large deformations of the free surface, a very small density ratio between gas and liquid, and a potential dewetting of the rotating disk. Under these constraints, experimental parameters, namely liquid viscosity, disk angular speed and container size, have been selected to allow for the companion numerical study to be carried out. This is achieved by keeping the Reynolds number within the range 1200-3800. The original contribution of this paper is an in-depth comparison between both approaches for a density ratio germane to the usual air-water system. LDV measurements have been carried for the first time in this configuration for spin-up and permanent regimes.

Concerning spin-up, we find that the characteristic time to reach a permanent regime is of the order of the Ekman time scale $\tau_{E} \sim R e^{1 / 2}$. The facts that the system is started from rest (thus far from a linear spin-up), that the side walls are fixed and that a free surface is present, do not seem to have much influence. This interesting finding is likely to be also valid for larger Reynolds number flows.

Concerning the permanent regime, we observe that the surface deviation $\bar{h}(r)-G$ scales linearly with the Froude number. Surprisingly, the scaling holds for large surface deformations, even when the disk is dewetted (except of course in the dewetted region). Results show the change of the flow structure as dewetting occurs. As Fr increases, the central solid-body rotation disappears, which corroborates the conjecture of theoretical models [16]. Numerical simulations also provide the description of boundary and internal layers inside which the meridional circulation takes place. For the largest surface deformations, the surface layer and the disk boundary layer are connected and most of the circulation flows directly from the surface layer to disk layer.

Throughout this work, numerical simulations and experiments are carried out and emphasis is placed on comparisons. Both numerical and experimental approaches are precise enough to achieve excellent agreement, but also to learn from the observed discrepancies. In particular, we show that simulations using two different codes overestimate the azimuthal velocity measured experimentally at the core periphery. The issue of modelling the free surface using free-slip boundary conditions has already been raised in [24] for $F r=0$. We 
herein confirm this point as azimuthal velocity measurements differ from numerical results close to the free surface, but agree elsewhere. This is still an open question, however it is shown here, by precisely measuring the surface height, that it has a limited impact on the shape of the free surface. Another phenomenon is captured by comparing experimental results from two distinct experimental set-ups at same Froude number but different Reynolds numbers. For the higher Re, a bulk instability develops soon after the spin-up is completed. This instability modifies the mean permanent state and shifts the mean free surface, but, again, the impact is relatively small. We thus conjecture that the incipience of rotating polygons, as a surface-wave instability, is decoupled from that of bulk instabilities. The temporally averaged flow studied in this paper is then thought to be a good candidate as a base flow to study the emergence of rotating polygons.

Last, apart from the meniscus, the simplified treatment of the triple line (see boundary conditions for the level-set distance function in section 4 of the appendix) permits to satisfactorily reproduce the shape of the interface. It also fully captures the dynamics during the spin-up, including when dewetting occurs. This is coherent with the fact that the Weber number is large (here, larger than 390 for all cases).

As a first perspective to this paper, a systematic study of the onset of bulk instabilities for a deformed interface may be undertaken, e.g. through linear analysis. We conjecture that their physical mechanism is not changed if surface deformation is moderate, but, based on the results of section IV, dewetting probably interferes with their occurrence. Another perspective is the quantitative prediction of the linear instability of the base flow described herein, leading to rotating polygons, including dewetted cases. The response of such realistic flow solutions with large interface deformations, including dewetted cases, to three-dimensional perturbations remains to be investigated. Comparisons with experimental findings will permit to identify the nature of the bifurcation and to understand the hysteresis previously observed in this system [6].

\section{ACKNOWLEDGMENTS}

This work was supported by the French Agence Nationale de la Recherche under the ANR ETAE Project No. ANR-16-CE08-0011. The authors would like to acknowledge Kronos for supplying us with Titanium dioxide particles, Antoine Faugaret, François Lusseyran, 
Wietze Herreman, Caroline Nore, Guangyang Cui and the technical team at LIMSI for their help on the experimental facility or/and fruitful discussions. The authors would like to thank an anonymous referee for constructive comments.

\section{Appendix: Numerical methodology for Sunfluidh}

The numerical methods used in SUNFLUIDH are presented here with a focus on the treatment needed by the sharp variations of physical quantities like mass density and dynamical viscosity, handled with a level-set formulation [42]. Hereafter, $\delta t$ stands for the time step, superscript $(n)$ for the time index such that $t^{(n)}=n \delta t, \boldsymbol{x}$ for the position vector, $\boldsymbol{e}_{i}$ for the $i^{\text {th }}$ unit basis vector and $Q_{i}$ for the $i^{t h}$ component of any vectorial quantity $\boldsymbol{Q}$, that is $Q_{i}=\boldsymbol{Q} \cdot \boldsymbol{e}_{i}$

For each time step, the algorithm can be decomposed into 3 stages: $(i)$ the time advance of the level-step function, from which the mass density and the dynamical viscosity are derived, (ii) the computation of the surface tension and buoyancy force, (iii) the time advance of the velocity components, which involves a prediction and a projection step.

\section{Solving for mass density and dynamical viscosity with the level-set method}

Following Osher \& Sethian [42] and Sussman \& Osher ([43]), the level-set function $\phi$ is chosen as a signed distance from the interface, thus being 0 at the interface. Let variables $\rho_{i}$ and $\mu_{i}$ denote the density and viscosity of the fluid $i(i=1$ or 2 ). The spatial distribution of $\rho$ and $\mu$ is given by the relations:

$$
\rho(\phi)=\rho_{1}+\left(\rho_{2}-\rho_{1}\right) H^{\epsilon}(\phi), \quad \mu(\phi)=\mu_{1}+\left(\mu_{2}-\mu_{1}\right) H^{\epsilon}(\phi)
$$

where $H^{\epsilon}$ is a regularized Heaviside function:

$$
H^{\epsilon}(\phi)= \begin{cases}0 & \text { if } \phi<-\epsilon \\ \frac{1}{2}+\frac{\phi}{2 \epsilon}+\frac{1}{2 \pi} \sin \left(\frac{\pi \phi}{\epsilon}\right) & \text { if }|\phi| \leq \epsilon \\ 1 & \text { if } \phi>\epsilon\end{cases}
$$

and $\epsilon$ is a regularization length scale of the same order of magnitude as the cell size. 
For an incompressible flow, the governing equation for $\phi$ in conservative form reads as:

$$
\frac{\partial \phi}{\partial t}+\nabla \cdot(\phi \mathbf{V})=0
$$

When time advance is applied, the resulting function $\tilde{\phi}^{(n+1)}$ does not satisfy the property $\|\nabla \phi\|=1$ characteristic of a distance function, as the velocity field is, in general, nonuniform. Yet, enforcing this property is particularly important at the vicinity of the interface as the control of the numerical thickness of the interface to a fixed value of $2 \epsilon$ uses a metric based on the $\phi$ function. This point is crucial to accurately evaluate the vector normal to the interface and thus surface tension.

The preservation of the above property is carried out with the redistancing method [43], which consists in correcting the solution $\tilde{\phi}^{(n+1)}$ by using the asymptotic solution of a relaxation equation

$$
\frac{\partial \phi}{\partial t^{\prime}}=\operatorname{sign}(\phi)(1-\|\nabla \phi\|)
$$

starting from the initial condition $\tilde{\phi}^{(n+1)}$. The location of the interface obtained by the relaxation (A.4) is the same as the location yielded by equation (A.3). In practice, only a few relaxation steps of (A.4) are sufficient to recover $\|\nabla \phi\|=1$ close to the interface. Equations (A.3) and (A.4) are both solved in time and space with high-order schemes. We here choose an explicit $3^{\text {rd }}$ order Runge-Kutta [44] scheme and a $5^{\text {th }}$ order WENO scheme [45] in order to ensure the stability of the solution.

\section{Solving the surface tension from the level set method}

The surface tension $\boldsymbol{S}=W e^{-1} \kappa \delta_{S} \boldsymbol{n}$ is estimated with the Continuum Surface Force (CSF) method [46, 47], which consists in approximating the tension at the surface by a body force. This involves a regularized Dirac function $\delta^{\epsilon}(\phi)=\mathrm{d} H^{\epsilon}(\phi) / \mathrm{d} \phi$. The surface tension is then expressed using function $\phi$ :

$$
\boldsymbol{S}=\frac{1}{W e} \kappa \delta^{\epsilon}(\phi) \frac{\nabla \phi}{\|\nabla \phi\|}, \quad \text { with } \mathbf{n}=\frac{\nabla \phi}{\|\nabla \phi\|} \quad \text { and } \quad \kappa=\nabla \cdot \mathbf{n} .
$$

The computation of curvature $\kappa$ is particularly sensitive to the numerical truncation errors. For this reason, high-order schemes are chosen to solve equations (A.3) and (A.4). When surface tension is computed directly via a discrete formulation of relations (A.5), numerical spurious or parasitic currents are generated in the vicinity of the surface (see [48] for a survey 
on this topic). We have chosen to compute the curvature by an accurate method proposed by Coquerelle et al. [49], where the curvature at each discrete point involved in the CSF method is estimated from that defined at the closest surface point.

\section{Solving the Navier-Stokes equations with the projection method}

The Navier-Stokes equations are solved by means of a projection method [50] in incremental form [38]. Two stages are necessary: a prediction step, whereby the velocity $\tilde{\boldsymbol{V}}^{(n+1)}$ at time $t^{(n+1)}$ is estimated, and a projection step which computes a potential field in order to obtain the pressure field $p^{(n+1)}$ and correct $\tilde{\boldsymbol{V}}^{(n+1)}$ into a divergence-free velocity $\boldsymbol{V}^{(n+1)}$.

In order to facilitate the presentation of numerical methods, the equation set (2) presented in section II B can be recast as

$$
\begin{aligned}
\nabla \cdot \boldsymbol{V} & =0, \\
\frac{\partial \boldsymbol{V}}{\partial t} & =\frac{1}{\rho}(\boldsymbol{C}-\nabla p+\boldsymbol{T}+\boldsymbol{B}+\boldsymbol{S}),
\end{aligned}
$$

where quantities $\boldsymbol{C}, \boldsymbol{T}, \boldsymbol{B}$ and $\boldsymbol{S}$ respectively refer to the convection term, the viscous stress, the buoyancy force, and the surface tension term:

$$
\boldsymbol{C}=-\rho(\boldsymbol{V} \cdot \nabla) \boldsymbol{V}, \quad \boldsymbol{T}=\frac{1}{R e} \nabla \cdot\left[\mu\left(\nabla \boldsymbol{V}+\nabla \boldsymbol{V}^{T}\right)\right], \quad \boldsymbol{B}=-\frac{1}{F r} \rho \boldsymbol{e}_{z}, \quad \boldsymbol{S}=\frac{1}{W e} \kappa \delta_{S} \boldsymbol{n} .
$$

Each component of equation (A.6b) is discretized following a finite-volume approach on a staggered structured grid. Scalar quantities and velocity components are respectively located at the center of the cells and at the center of cell faces. The spatial discretization is carried out using a second order centered scheme. The backward time discretization is also secondorder accurate. An implicit discretization is applied to the viscous stress term in order to enhance the numerical stability with respect to the time step. As velocity components $V_{i}$ are solved independently, the implicitation only concerns terms depending on $V_{i}$. So, each viscous stress component is split in two parts, $\widetilde{T}_{i}$ depending on $\tilde{V}_{i}$, and $T e_{i}$ depending on the other velocity components:

$$
T_{i}=\widetilde{T}_{i}+T e_{i} .
$$

Explicit terms, namely the viscous stress part $T e_{i}$ and the convection flux $C_{i}$, are estimated at time $t^{(n+1)}$ using a linear extrapolation of the known values at times $t^{(n)}$ and $t^{(n-1)}$ :

$$
E_{i}^{(n+1)}=2 E_{i}^{(n)}-E_{i}^{(n-1)} \text { with } \quad E_{i}=C_{i}+T e_{i} .
$$


The buoyancy $B_{i}^{(n+1)}$ and the surface tension $S_{i}^{(n+1)}$ are obtained from the density and the interface position already known at time $t^{(n+1)}$ thanks to the level-set method. The pressure gradient term is evaluated at time $t^{(n)}$.

The time-discretized equation for each component reads:

$$
\frac{3 \tilde{V}_{i}^{(n+1)}-4 V_{i}^{(n)}+V_{i}^{(n-1)}}{2 \delta t}=\frac{1}{\rho^{(n+1)}}\left(E_{i}^{(n+1)}+B_{i}^{(n+1)}+S_{i}^{(n+1)}-\nabla p^{(n)} \cdot \boldsymbol{e}_{i}+\widetilde{T i}_{i}^{(n+1)}\right) .
$$

After introducing the (unknown) velocity increment $\delta \tilde{V}_{i}^{(n+1)}=\tilde{V}_{i}^{(n+1)}-V_{i}^{(n)}$, equation (A.9) leads to the Helmholtz equation:

$$
\left(1-\frac{2 \delta t}{3 \rho^{(n+1)}} \Theta\right) \delta \tilde{V}_{i}^{(n+1)}=R_{i}
$$

where the right-hand side $R_{i}$ gathers all the known terms:

$$
R_{i}=\frac{2 \delta t}{3 \rho^{(n+1)}}\left(E_{i}^{(n+1)}+B_{i}^{(n+1)}+S_{i}^{(n+1)}+\Theta V_{i}^{(n)}-\nabla p^{(n)} \cdot \boldsymbol{e}_{i}\right)+\frac{1}{3}\left(V_{i}^{(n)}-V_{i}^{(n-1)}\right)
$$

and $\Theta$ is the linear operator such that $\Theta \tilde{V}_{i}=\widetilde{T}_{i}$. Once discretized in space, equations (A.10) are solved by means of an Alternating Direction Implicit method [51], which leads to the estimated velocity $\tilde{V}_{i}$ (prediction step).

Incompressibility is enforced by correcting $\tilde{\boldsymbol{V}}$. Using a projection method [50] under the incremental formulation [38], this leads, for cases with large density variations, to solve a Poisson-like equation:

$$
\nabla \cdot\left(\frac{1}{\rho^{(n+1)}} \nabla \Phi\right)=\frac{\nabla \cdot \tilde{\boldsymbol{V}}^{(n+1)}}{\delta t} .
$$

The solution $\Phi$ is computed using Successive Over-Relaxations coupled with an algebraic multi-grid approach [52] for convergence acceleration. The pressure field and the divergencefree velocity field $\boldsymbol{V}^{(n+1)}$ at time $t^{(n+1)}$ are deduced (correction step) through:

$$
\begin{aligned}
& p^{(n+1)}=p^{(n)}+\Phi, \\
& \boldsymbol{V}^{(n+1)}=\tilde{\boldsymbol{V}}^{(n+1)}-\frac{2}{3} \delta t \nabla \Phi .
\end{aligned}
$$

\section{Boundary conditions}

Equation governing the level-set distance function (A.3): the boundary conditions commonly used for such equation is a zero normal derivative for all boundaries. This satisfies the symmetry condition at the axis (for an axisymmetric configuration) and is kept. At the 
walls, we however choose another boundary condition: the normal second derivative of the distance function is set to zero. This approach tends to relax the constraint on the wetting angle, which would always lead to the nonphysical value $\pi / 2$ on the disk if the normal first derivative was set to zero. The differences nevertheless remain moderate as surface tension is very small here.

Helmholtz equations (A.10): for the velocity components at the walls, Dirichlet conditions are imposed, which correspond to the impermeability and no-slip (or driven) conditions. At the axis, axisymmetry imposes symmetry boundary conditions: zero normal derivative for the axial velocity component and zero radial and azimuthal velocity components. At the top boundary, stress-free conditions are enforced through symmetry boundary conditions: zero axial velocity component and zero normal derivative for radial and azimuthal velocity components.

Poisson equation (A.12): the conditions at the domain ends are zero normal derivative of $\Phi$, as usually done for the projection step, when the value of the normal velocity component is known.

[1] G. H. Vatistas, A note on liquid vortex sloshing and Kelvin's equilibria, J. Fluid Mech. 217, $241(1990)$.

[2] T. R. N. Jansson, M. P. Haspang, K. H. Jensen, P. Hersen, and T. Bohr, Polygons on a rotating fluid surface, Phys. Rev. Lett. 96, 174502 (2006).

[3] T. Suzuki, M. Iima, and Y. Hayase, Surface switching of rotating fluid in a cylinder, Phys. Fluids 18, 101701(4) (2006).

[4] R. Bergmann, L. Tophøj, T. A. M. Homan, P. Hersen, A. Andersen, and T. Bohr, Polygon formation and surface flow on a rotating fluid surface, J. Fluid Mech. 679, 415 (2011).

[5] K. Iga, S. Yokota, S. Watanabe, T. Ikeda, H. Niino, and N. Misawa, Various phenomena on a water vortex in a cylindrical tank over a rotating bottom, Fluid Dyn. Res. 46, 031409 (2014).

[6] B. Bach, E. Linnartz, M. Vested, A. Andersen, and T. Bohr, From Newtons bucket to rotating polygons: experiments on surface instabilities in swirling flows, J. Fluid Mech. 759, 386 (2014).

[7] M. Iima and Y. Tasaka, Dynamics of flow structures and surface shapes in the surface switching of rotating fluid, J. Fluid Mech. 789, 402 (2016). 
[8] H. A. Abderrahmane, M. Fayed, H. D. Ng, and G. H. Vatistas, The effect of viscosity on the rotating waves and polygonal patterns within a hollow vortex core, Exp. Therm. Fluid Sci. 87, 104 (2017).

[9] A. Spohn, M. Mory, and E. Hopfinger, Observations of vortex breakdown in an open cylindrical container with a rotating bottom, Exp. Fluids 14, 70 (1993).

[10] D. L. Young, H. J. Sheen, and T. Y. Hwu, Period-doubling route to chaos for a swirling flow in an open cylindrical container with a rotating disk, Exp. Fluids 18, 389 (1995).

[11] A. H. Hirsa, J. M. Lopez, and R. Miraghaie, Symmetry breaking to a rotating wave in a lid-driven cylinder with a free surface: Experimental observation, Phys. Fluids 14, 29 (2002).

[12] S. Poncet and M. Chauve, Shear-layer instability in a rotating system, J. Flow Visual. Image Process. 14, 85 (2007).

[13] J. M. Lopez and F. Marques, Mode competition between rotating waves in a swirling flow with reflection symmetry, J. Fluid Mech. 507, 265 (2004).

[14] L. Kahouadji, L. Martin Witkowski, and P. Le Quéré, Seuils de stabilité pour un écoulement à surface libre engendré dans une cavité cylindrique tournante à petit rapport de forme, Mécanique et Industries 11, 339 (2010).

[15] L. Tophøj, J. Mougel, T. Bohr, and D. Fabre, Rotating polygon instability of a swirling free surface flow, Phys. Rev. Lett. 110, 194502 (2013).

[16] D. Fabre and J. Mougel, Generation of three-dimensional patterns through wave interaction in a model of free surface swirling flow, Fluid Dyn. Res. 46, 061415 (2014).

[17] K. Iga, S. Yokota, S. Watanabe, T. Ikeda, H. Niino, and N. Misawa, Axisymmetric flow in a cylindrical tank over a rotating bottom. Part II. Deformation of the water surface and experimental verification of the theory, Fluid Dyn. Res. 49, 065501 (2017).

[18] J. Mougel, D. Fabre, L. Lacaze, and T. Bohr, On the instabilities of a potential vortex with a free surface, J. Fluid Mech. 824, 230 (2017).

[19] P. T. Brady, M. Herrmann, and J. M. Lopez, Two-fluid confined flow in a cylinder driven by a rotating endwall, Phys. Rev. E 85, 016308 (2012).

[20] L. Carrión, M. A. Herrada, and V. N. Shtern, Topology changes in a water-oil swirling flow, Phys. Fluids 29, 032109 (2017).

[21] S. Fujimoto and Y. Takeda, Topology changes of the interface between two immiscible liquid layers by a rotating lid, Phys. Rev. E 80, 015304 (2009). 
[22] M. A. Herrada, V. N. Shtern, and J. M. López-Herrera, Off-axis vortex breakdown in a shallow whirlpool, Phys. Rev. E 87, 063016(8) (2013).

[23] L. Kahouadji and L. Martin Witkowski, Free surface due to a flow driven by a rotating disk inside a vertical cylindrical tank: Axisymmetric configuration, Phys. Fluids 26, 072105(17) (2014).

[24] W. Yang, I. Delbende, Y. Fraigneau, and L. Martin Witkowski, Axisymmetric rotating flow with free surface in a cylindrical tank, J. Fluid Mech. 861, 796 (2019).

[25] E. R. Benton and A. Clark Jr, Spin-up, Ann. Rev. Fluid Mech. 6, 257 (1974).

[26] P. Duck and M. Foster, Spin-up of homogeneous and stratified fluids, Ann. Rev. Fluid Mech. 33, 231 (2001).

[27] E. H. Wedemeyer, The unsteady flow within a spinning cylinder, J. Fluid Mech. 20, 383 (1964).

[28] W. B. Watkins and R. G. Hussey, Spin-up from rest: Limitations of the Wedemeyer model, Phys. Fluids 16, 1530 (1973).

[29] W. B. Watkins and R. G. Hussey, Spin-up from rest in a cylinder, Phys. Fluids 20, 1596 (1977).

[30] A. Warn-Varnas, W. W. Fowlis, S. Piacsek, and S. M. Lee, Numerical solutions and laserdoppler measurements of spin-up, J. Fluid Mech. 85, 609 (1978).

[31] J. M. Hyun, F. Leslie, W. W. Fowlis, and A. Warn-Varnas, Numerical solutions for spin-up from rest in a cylinder, J. Fluid Mech. 127, 263 (1983).

[32] H. Greenspan and L. Howard, On a time-dependent motion of a rotating fluid, J. Fluid Mech. 17, 385 (1963).

[33] J. O'Donnell and P. F. Linden, Free-surface effects on the spin-up of fluid in a rotating cylinder, J. Fluid Mech. 232, 439 (1991).

[34] F. Yan and B. Farouk, Numerical simulations of flows inside a partially filled centrifuge, J. Fluids Eng. 125, 1033 (2003).

[35] K. Iga, Axisymmetric flow in a cylindrical tank over a rotating bottom. Part I. Analysis of boundary layers and vertical circulation, Fluid Dyn. Res. 49, 065502 (2017).

[36] N. Cheng, Formula for the viscosity of a Glycerol-Water mixture, Ind. Eng. Chem. Res. 47, 3285 (2008).

[37] A. Przadka, B. Cabane, V. Pagneux, A. Maurel, and P. Petitjeans, Fourier transform pro- 
filometry for water waves: how to achieve clean water attenuation with diffusive reflection at the water surface?, Exp. Fluids 52, 519 (2012).

[38] K. Goda, A multistep technique with implicit difference schemes for calculating two- or threedimensional cavity flows, J. Comput. Phys. 30, 76 (1979).

[39] Movie of the spin-up corresponding to figure 4.

[40] J. M. Lopez, F. Marques, A. H. Hirsa, and R. Miraghaie, Symmetry breaking in free-surface cylinder flows, J. Fluid Mech. 502, 99 (2004).

[41] Movie of the spin-up corresponding to figures 12 and 13. Red curves refer to measurements of surface height while dashed thick black curves refer to numerical ones. The numerical azimuthal velocity and stream function are selected as explained in the caption of figure 13.

[42] S. Osher and J. A. Sethian, Fronts propagating with curvature-dependent speed : Algorithms based on Hamilton-Jacobi formulations, J. Comput. Phys. 79, 12 (1988).

[43] M. Sussman, P. Smereka, and S. Osher, A level set approach for computing solutions to incompressible two-phase flow, J. Comput. Phys. 114, 146 (1994).

[44] S. Gottlieb and C. W. Shu, Total variation diminishing runge-kutta schemes, Math. Comp. 67, 73 (1998).

[45] G.-S. Jiang and D. Peng, Weighted ENO schemes for Hamilton-Jacobi equations, SIAM J. Sci. Comput. 21, 2126 (2000).

[46] J. U. Brackbill, D. B. Kothe, and C. Zemach, A continuum method for modeling surface tension, J. Comput. Phys. 100, 335 (1992).

[47] Y.-C. Chang, T. Hou, B. Merriman, and S. Osher, A level set formulation of Eulerian interface capturing methods for incompressible fluid flows, J. Comput. Phys. 124, 449 (1996).

[48] S. Popinet, Numerical models of surface tension, Ann. Rev. Fluid Mech. 50, 49 (2018).

[49] M. Coquerelle and S. Glockner, A fourth-order accurate curvature computation in a level set framework for two-phase flows subjected to surface tension forces, J. Comput. Phys. 305, 838 (2016).

[50] J. L. Guermond, P. D. Minev, and J. Shen, An overview of projection methods for incompressible flows, Comp. Meth. Appl. Mech. Eng. 195, 6011 (2006).

[51] D. W. Peaceman and H. H. Rachford, The numerical solution of parabolic and elliptic differential equations, J. Soc. Ind. Appl. Math. 3, 28 (1955).

[52] Y. Saad, Iterative Methods for Sparse Linear Systems, 2nd ed. (Society for Industrial and 
Applied Mathematics, 2003). 